\title{
Alternating Tree Automata with Qualitative Semantics
}

\author{
RAPHAËL BERTHON, Université libre de Bruxelles, Belgium \\ NATHANAËL FIJALKOW, CNRS \& LaBRI, France \\ EMMANUEL FILIOT, Université libre de Bruxelles, Belgium \\ SHIBASHIS GUHA, Université libre de Bruxelles, Belgium \\ BASTIEN MAUBERT, Università degli Studi di Napoli “Federico II", Italy \\ ANIELLO MURANO, Università degli Studi di Napoli "Federico II", Italy \\ LAURELINE PINAULT, Univ Lyon, CNRS, ENS de Lyon, UCB Lyon 1, LIP, France \\ SOPHIE PINCHINAT, Univ Rennes, CNRS, IRISA, France \\ SASHA RUBIN, University of Sydney, Australia \\ OLIVIER SERRE, Université de Paris, IRIF, CNRS, France
}

\begin{abstract}
We study alternating automata with qualitative semantics over infinite binary trees: alternation means that two opposing players construct a decoration of the input tree called a run, and the qualitative semantics says that a run of the automaton is accepting if almost all branches of the run are accepting. In this paper we prove a positive and a negative result for the emptiness problem of alternating automata with qualitative semantics.

The positive result is the decidability of the emptiness problem for the case of Büchi acceptance condition. An interesting aspect of our approach is that we do not extend the classical solution for solving the emptiness problem of alternating automata, which first constructs an equivalent non-deterministic automaton. Instead, we directly construct an emptiness game making use of imperfect information.

The negative result is the undecidability of the emptiness problem for the case of co-Büchi acceptance condition. This result has two direct consequences: the undecidability of monadic second-order logic extended with the qualitative path-measure quantifier, and the undecidability of the emptiness problem for alternating tree automata with non-zero semantics, a recently introduced probabilistic model of alternating tree automata.
\end{abstract}

CCS Concepts: • Theory of computation $\rightarrow$ Automata over infinite objects; Tree languages; Quantitative automata; Logic; Probabilistic computation.

Additional Key Words and Phrases: tree automata, $\omega$-regular conditions, almost-sure semantics

Authors' addresses: Raphaël Berthon, Université libre de Bruxelles, Bruxelles, Belgium, Raphael.Berthon@ulb.ac.be; Nathanaël Fijalkow, CNRS \& LaBRI, Bordeaux, France, Nathanael.Fijalkow@labri.fr; Emmanuel Filiot, Université libre de Bruxelles, Bruxelles, Belgium, efiliot@ulb.ac.be; Shibashis Guha, Université libre de Bruxelles, Bruxelles, Belgium, Shibashis. Guha@ulb.ac.be; Bastien Maubert, Università degli Studi di Napoli “Federico II”, DIETI, Naples, Italy, bastien.maubert@ gmail.com; Aniello Murano, Università degli Studi di Napoli "Federico II”, DIETI, Naples, Italy, murano@na.infn.it; Laureline Pinault, Univ Lyon, CNRS, ENS de Lyon, UCB Lyon 1, LIP, Lyon, France, Laureline.Pinault@ens-lyon.fr; Sophie Pinchinat, Univ Rennes, CNRS, IRISA, Rennes, France, Sophie.Pinchinat@irisa.fr; Sasha Rubin, University of Sydney, Sydney, Australia, rubin@forsyte.at; Olivier Serre, Université de Paris, IRIF, CNRS, Bâtiment Sophie Germain, Case courrier 7014, 8 Place Aurélie Nemours, Paris Cedex 13, 75205, France, Olivier.Serre@cnrs.fr.

Permission to make digital or hard copies of all or part of this work for personal or classroom use is granted without fee provided that copies are not made or distributed for profit or commercial advantage and that copies bear this notice and the full citation on the first page. Copyrights for components of this work owned by others than ACM must be honored. Abstracting with credit is permitted. To copy otherwise, or republish, to post on servers or to redistribute to lists, requires prior specific permission and/or a fee. Request permissions from permissions@acm.org.

(C) 2020 Association for Computing Machinery.

1529-3785/2020/12-ART \$15.00

https://doi.org/10.1145/nnnnnnn.nnnnnnn

ACM Trans. Comput. Logic, Vol. 1, No. 1, Article . Publication date: December 2020. 
ACM Reference Format:

Raphaël Berthon, Nathanaël Fijalkow, Emmanuel Filiot, Shibashis Guha, Bastien Maubert, Aniello Murano, Laureline Pinault, Sophie Pinchinat, Sasha Rubin, and Olivier Serre. 2020. Alternating Tree Automata with Qualitative Semantics. ACM Trans. Comput. Logic 1, 1 (December 2020), 24 pages. https://doi.org/10.1145/nnnnnnn.nnnnnn

\section{INTRODUCTION}

The study of tree-automata models can be organised by distinguishing three semantic features.

The first feature is the operational mode: deterministic, non-deterministic, universal, probabilistic, and alternating, are the most studied notions. Intuitively, in each case, an automaton reading an input tree (with labels on the nodes) constructs a decoration of this tree called a run, which is itself a tree. The run labels nodes of the tree by states respecting the local constraints imposed by the transition relation of the automaton. In the deterministic case, a state and a letter uniquely determine the labels at the level below in the run, hence there is a unique run. In the non-deterministic, universal, and alternating case, there may be several valid transitions at each node, yielding possibly several runs on a single tree. In the non-deterministic case we say that the tree is accepted if there exists an accepting run, i.e. the choices are existential. In the universal case, we say that the tree is accepted if all runs are accepting, i.e. the choices are universal. The alternating case unifies both previous cases by introducing existential and universal transitions.

The second feature is the branching semantics. The classical one says that a run is accepting if all its branches satisfy a given acceptance condition. We are concerned in this paper with the qualitative semantics, which is an alternative branching semantics introduced by Carayol, Haddad, and Serre [8]. The qualitative semantics says that a run is accepting if almost all its branches satisfy a given acceptance condition, in other words if by picking a branch uniformly at random it almost-surely satisfies the condition. The paper [8] showed that non-deterministic and probabilistic tree automata with qualitative semantics are both robust computational models with appealing algorithmic properties.

The third feature is the acceptance condition (on branches), with $\omega$-regular conditions such as Büchi and parity conditions being the most important for their tight connections to logical formalisms; see, e.g., [32].

One motivation for studying tree automata with qualitative semantics is to extend the deep connections between automata and monadic second-order logic (MSO) which hold for the classical semantics [29]. Indeed, the general goal is to construct decidable extensions of MSO over infinite trees; we review some of the efforts and results obtained in this direction. A (unary) generalised quantifier is of the form "the set of all sets $X$ that satisfy $\varphi$ has the property $C$ ", where $C$ is a property of sets. For instance, the ordinary existential quantifier $\exists X . \varphi$ corresponds to the property $C$ of being a non-empty set. More interestingly, the quantifier "there exist infinitely many $X$ such that $\varphi$ " corresponds to the property $C$ of being infinite. It turns out that certain cardinality quantifiers such as "there exist infinitely many $X$ " and "there exist continuum many $X$ " do not add expressive power to MSO over the infinite binary tree (in fact, they can be effectively eliminated) [2]. On the other hand, adding the generalised quantifier "the set of all sets $X$ satisfying $\varphi$ has Lebesgue-measure one" results in an undecidable theory [25]. A weaker version of this quantifier is "the set of paths of the tree that satisfy $\varphi$ has Lebesgue-measure one". Intuitively, this quantifier, written $\underset{\text { path }}{\forall}=1$, means that a random path almost-surely satisfies $\varphi$, where a random path is generated by repeatedly flipping a coin to decide whether to go left or right. It was proved in $[5,6]$ that adding the quantifier $\forall=1$ path to a restriction of MSO called "thin MSO" yields a decidable logic, but the decidability of $\mathrm{MSO}+\forall_{\text {path }}=1$ was left open in $[24,25]$. The emptiness problem for non-deterministic parity tree 
automata with qualitative semantics can easily be expressed using MSO $+\underset{\text { path }}{=1}$, as already observed in [25], and this is also the case for universal tree automata with qualitative semantics.

In this paper, we initiate the study of alternating automata with qualitative semantics, and focus on the emptiness problem. We present a positive result and a negative result that delimit a clear and sharp decidability frontier.

\section{Contributions}

The positive result is the decidability of the emptiness problem for the case of the Büchi acceptance condition (Theorem 3.7).

The usual roadmap for solving the emptiness problem for alternating automata is to first construct an equivalent non-deterministic automaton, and then to construct an emptiness game for the non-deterministic automaton, i.e., a game such that the first player wins if and only if the automaton is non-empty. This first step is an effective construction of an equivalent non-deterministic automaton, which in some cases is not possible, unknown, or computationally too expensive. In the case at hand the second situation arises: we do not know whether alternating automata with qualitative semantics can effectively be turned into equivalent non-deterministic ones. We remark that our undecidability result shows that there is no such effective construction for co-Büchi conditions (but there might be one for the Büchi conditions).

Here, instead, we develop a new approach which directly constructs an emptiness game for the alternating automaton. The emptiness game we construct uses imperfect information. Our construction extends the notion of blindfold games of Reif [30], used to check universality of nondeterministic automata over finite words. The key ingredient to proving the correctness of our imperfect information emptiness game is a new positionality result for stochastic Büchi games on certain infinite arenas (that we call chronological). To the best of our knowledge, very few positionality results are known in the literature that combine both stochastic features and infinite arenas; a notable exception is [23].

The negative result is the undecidability of the emptiness problem for the case of the co-Büchi acceptance condition. In fact, our main technical contribution (Theorem 4.2) is to establish the undecidability already for universal automata (a special subclass of alternating automata).

We establish this by a chain of reductions that consider various classes of automata (both on infinite words and trees). We initially resort to the known undecidability of the value 1 problem for probabilistic automata on finite words [20] to deduce the undecidability of the emptiness problem for simple probabilistic co-Büchi automata on infinite words (Proposition 4.1). Here, simple means that the transitions of the automaton only involve probabilities in $\left\{0, \frac{1}{2}, 1\right\}$. Then, we reduce the latter problem to the original emptiness problem for universal co-Büchi tree automata with qualitative semantics, hence proving our negative result. The correctness of this last reduction relies on particular properties of another class of automata, namely, probabilistic tree automata.

Our negative result has two interesting consequences: the undecidability of $\mathrm{MSO}+\mathrm{V}_{\text {path }}=1$, and of the emptiness problem for alternating tree automata with non-zero semantics, a model combining sure, almost-sure, and positive semantics and studied in [19].

\section{Related Work}

The study of automata with qualitative semantics was initiated in [8] with several decidability results. The first result is a polynomial-time algorithm entailing the decidability of the emptiness problem for non-deterministic parity tree automata with qualitative semantics [8], obtained through a polynomial reduction to the almost-sure problem for Markov decision processes (for 
which a polynomial-time algorithm is known from [12]). This reduction extends to probabilistic tree automata with qualitative semantics, showing an equivalence with partial-observation Markov decision processes. It is then used to prove the decidability of the emptiness problem for probabilistic Büchi tree automata with qualitative semantics [8].

Alternation was later considered by Fijalkow, Pinchinat, and Serre in [17] where the focus was on designing a novel emptiness checking procedure working directly on alternating automata, i.e. directly building an emptiness imperfect-information game without making use of the intermediate transformation to a non-deterministic automaton: this was successfully applied to classical alternating parity tree automata as well as to alternating Büchi tree automata with qualitative semantics (see Theorem 3.7).

This line of work was pursued using the related model of non-zero automata. The first decidability result was obtained for the subclass of zero automata [6], yielding the decidability of the thin restriction of $\mathrm{MSO}+\forall_{\text {path }}^{=1}$. A second decidability result concerned the class of alternating zero automata [19], restricting the abilities of the second player. This latter result is applied to solve the satisfiability problem of a probabilistic extension of CTL*. The general case of non-zero automata was left open. We close it negatively (thanks to our negative result) since alternating tree automata with non-zero semantics subsume universal tree automata with qualitative semantics.

A side result in [17] states the undecidability of the emptiness problem for alternating co-Büchi automata with qualitative semantics. The proof, not given in the conference proceedings, is rather sketchy in the full version [18]. The proof we give here (Theorem 4.2) follows the same lines but clarifies a technical loophole in the original proof. Indeed, the last reduction requires the undecidability of the emptiness problem for probabilistic co-Büchi simple automata over infinite words, where simple means that the transitions probabilities are either $0, \frac{1}{2}$, or 1 . The undecidability result was known only for general automata, while we refine it for the simple ones, thus filling in the gap of the undecidability proof in the full version [18].

More recently, Berthon et al. [3] proved the slightly weaker undecidability result that emptiness is undecidable for universal parity tree automata with qualitative semantics. Although their proof follows the same lines as [18], the result is weaker because they need a stronger acceptance condition to obtain simple automata and prove the correctness of the original reduction. Still, their result is strong enough to entail the undecidability of $\mathrm{MSO}+\underset{\text { path }}{=1}$, the main contribution of their work.

There is another proof of the undecidability of $\mathrm{MSO}+\mathrm{V}_{\text {path }}^{=1}$, obtained independently and at the same time as [3] by Bojańczyk, Kelmendi, and Skrzypczak [7]. Their proof technique is very different from ours: they obtain undecidability by a direct encoding of two-counter machines into the logic. However, the core technical part of the paper is not the reduction from counter machines (which is nevertheless tricky), but a crucial technical lemma used to encode runs of counter machines and to prove the correctness of the reduction ${ }^{1}$. The proof of this lemma is involved: it mostly relies on tools (such as asymptotic behaviours of vector sequences) previously used to show undecidability of $\mathrm{MSO}+\mathrm{U}$ logic over infinite words. We remark that $\mathrm{MSO}+\underset{\text { path }}{\forall}=1$ is known as $\mathrm{MSO}+\nabla$ in [7].

\section{Organisation of the Paper}

Section 2 presents the different classes of automata used for our main undecidability result, relying on Markov chains as a unifying notion to define acceptance by these different automata. Section 3

\footnotetext{
${ }^{1}$ More precisely, the lemma states that for a set $D$ of pairwise disjoint finite paths in the infinite binary tree called intervals, there is an $\mathrm{MSO}+\forall_{\text {path }}^{=1}$ formula that, when true at the root of the infinite binary tree, is equivalent to having with probability 1 , a branch $\pi$ and some integer $\ell$ such that with finitely many exceptions, if an interval intersects $\pi$ then it is of length $\ell$.
} 
gives our decidability result for alternating Büchi tree automata. Section 4 is about our undecidability result for universal co-Büchi tree automata, while Section 5 presents its consequences for $\mathrm{MSO}+\underset{\text { path }}{\forall=1}($ Section 5.1) and for alternating automata with non-zero semantics (Section 5.2).

\section{PRELIMINARIES}

Throughout the paper we implicitly fix a finite alphabet $\Sigma$. We denote by $\Sigma^{*}$ the set of finite words over $\Sigma$ and by $\Sigma^{\omega}$ the set of infinite words over $\Sigma$. We let $\varepsilon$ denote the empty word, and for a word $u \in \Sigma^{*},|u|$ denotes its length. Finally, we write $\Sigma^{k}$ for the set of words over $\Sigma$ of length $k$.

The infinite binary tree is $\{0,1\}^{*}$, elements of $\{0,1\}^{*}$ are called its nodes, and elements of $\{0,1\}^{\omega}$ are called its (infinite) branches. For a finite alphabet $\Sigma$, a $\Sigma$-tree is a function $t:\{0,1\}^{*} \rightarrow$ $\Sigma$ and we write Trees $(\Sigma)$ for the set of $\Sigma$-trees. For a branch $b=b_{1} b_{2} \cdots \in\{0,1\}^{\omega}$ we denote by $t[b]=t(\varepsilon) t\left(b_{1}\right) t\left(b_{1} b_{2}\right) t\left(b_{1} b_{2} b_{3}\right) \cdots \in \Sigma^{\omega}$ the infinite word read in $t$ along the branch $b$.

A distribution over a set $Q$ is a function $\delta: Q \rightarrow[0,1]$ such that $\sum_{q \in Q} \delta(q)=1$. Any distribution $\delta$ considered in the paper is implicitly assumed to have a finite support, i.e. $\{q \in Q \mid \delta(q) \neq 0\}$ is finite. For $Q^{\prime} \subseteq Q$, we write $\sum_{q \in Q^{\prime}} p_{q} \cdot q$ for the distribution that assigns probability $p_{q}$ to $q \in Q^{\prime}$ and 0 to $q \in Q \backslash Q^{\prime}$. For example, $\frac{1}{2} q_{1}+\frac{1}{2} q_{2}$ is the distribution $\delta$ such that $\delta\left(q_{1}\right)=\delta\left(q_{2}\right)=\frac{1}{2}$, unless $q_{1}=q_{2}$ in which case $\delta\left(q_{1}\right)=\delta\left(q_{2}\right)=1$, and $\delta(q)=0$ for every other element $q$. The set of distributions over $Q$ is denoted $\mathcal{D}(Q)$.

A Markov chain $\mathcal{M}=\left(S, s_{i n}, T\right)$ is given by a possibly infinite set of states $S$, an initial state $s_{\text {in }} \in S$, and a probability transition function $T: S \rightarrow \mathcal{D}(S)$. An (infinite) path in $\mathcal{M}$ is an infinite sequence of states $s_{0} s_{1} s_{2} \ldots \in S^{\omega}$ such that $s_{0}=s_{i n}$ and $T\left(s_{i}\right)\left(s_{i+1}\right)>0$ for every $i \geq 0$. A cone is a set of paths of the form $u \cdot S^{\omega}$ for some $u \in S^{*}$. Now, consider the $\sigma$-algebra over paths in $\mathcal{M}$ built from the set of cones. Then, a classical way to equip this $\sigma$-algebra with a probability measure $P$ is to recursively define it on the set of cones as follows:

$$
P\left(s_{0} s_{1} \cdots s_{k} \cdot S^{\omega}\right)= \begin{cases}1 & \text { if } k=0 \\ P\left(s_{0} \cdots s_{k-1} \cdot S^{\omega}\right) \cdot T\left(s_{k-1}\right)\left(s_{k}\right) & \text { otherwise }\end{cases}
$$

and then to extend it (uniquely) to the $\sigma$-algebra thanks to Carathéodory's extension theorem (we refer the reader to Reference [27] for more details on this classical construction).

When needed, for a given length $k$, we also see $P$ as a probability measure on paths of length $k$ (i.e. elements in $S^{k}$ ) by defining the probability measure of $u \in S^{k}$ as the probability of the cone $u \cdot S^{\omega}$.

\subsection{Two-Player Perfect-Information Stochastic Games}

A graph is a pair $G=(V, E)$ where $V$ is a (possibly infinite) set of vertices and $E \subseteq V \times V$ is a set of edges. For every vertex $v$, let $E(v)=\{w \mid(v, w) \in E\}$, and say that $v$ is a dead-end if $E(v)=\emptyset$. In the rest of the paper, we only consider graphs of finite out-degree, i.e. such that $|E(v)|$ is finite for every vertex $v \in V$, and without dead-ends.

A (turn-based) stochastic arena is a tuple $\mathcal{G}=\left(G, V_{E}, V_{A}, V_{R}, \delta, v_{\text {in }}\right)$ where $G=(V, E)$ is a graph, $\left(V_{E}, V_{A}, V_{R}\right)$ is a partition of the vertices among two players, Éloïse and Abélard, and an extra player Random, $\delta: V_{R} \rightarrow \mathcal{D}(V)$ is a map such that for all $v \in V_{R}$ the support of $\delta(v)$ is included in $E(v)$, and $v_{\text {in }} \in V$ is an initial vertex. In a vertex $v \in V_{E}$ (resp. $v \in V_{A}$ ) Éloïse (resp. Abélard) chooses a successor vertex from $E(v)$, and in a random vertex $v \in V_{R}$, a successor vertex is chosen according to the probability distribution $\delta(v)$. A play $\lambda=v_{0} v_{1} v_{2} \cdots$ is an infinite sequence of vertices starting from the initial vertex, i.e. $v_{0}=v_{i n}$, and such that, for every $k \geq 0, v_{k+1} \in E\left(v_{k}\right)$ if $v_{k} \in V_{E} \cup V_{A}$ and $\delta\left(v_{k}\right)\left(v_{k+1}\right)>0$ if $v_{k} \in V_{R}$. A history is a finite prefix of a play. 
A (pure $\left.{ }^{2}\right)$ strategy for Éloïse is a function $\sigma_{E}: V^{*} \cdot V_{E} \rightarrow V$ such that for every history $\lambda \cdot v \in$ $V^{*} \cdot V_{E}$ one has $\sigma_{E}(\lambda \cdot v) \in E(v)$. Strategies of Abélard are defined likewise, and usually denoted $\sigma_{A}$.

A play $\lambda=v_{0} v_{1} v_{2} \ldots$ is consistent with a pair of strategies $\left(\sigma_{E}, \sigma_{A}\right)$ for Éloïse and Abélard if the players always choose their move according to their strategy. Formally, for all $k \geq 0$ the following should hold: if $v_{k}$ is controlled by Éloïse then $v_{k+1}=\sigma_{E}\left(v_{0} \ldots v_{k}\right)$ and if it is controlled by Abélard then $v_{k+1}=\sigma_{A}\left(v_{0} \ldots v_{k}\right)$. The set of plays consistent with $\left(\sigma_{E}, \sigma_{A}\right)$ is denoted Plays $\sigma_{\sigma_{E}, \sigma_{A}}^{\mathcal{G}}$, and a history is consistent with $\left(\sigma_{E}, \sigma_{A}\right)$ if it is the finite prefix of some play in Plays ${ }_{\sigma_{E}, \sigma_{A}} \mathcal{G}$.

In order to equip the set Plays ${ }_{\sigma_{E}, \sigma_{A}}^{\mathcal{G}}$ with a probability measure, we define the following Markov chain $\mathcal{M}_{\sigma_{E}, \sigma_{A}}^{\mathcal{G}}$ : its set of states is the set of histories consistent with $\left(\sigma_{E}, \sigma_{A}\right)$, its initial state is $v_{i n}$, and its probability transition function $T$ is defined by

$$
T(\lambda \cdot v)= \begin{cases}\lambda \cdot v \cdot \sigma_{E}(\lambda \cdot v) & \text { if } v \in V_{E} \\ \lambda \cdot v \cdot \sigma_{A}(\lambda \cdot v) & \text { if } v \in V_{A} \\ \sum_{v^{\prime} \in E(v)} \delta(v)\left(v^{\prime}\right) \lambda \cdot v \cdot v^{\prime} & \text { if } v \in V_{R}\end{cases}
$$

Then, the set $\operatorname{Plays}_{\sigma_{E}, \sigma_{A}}^{\mathcal{G}}$ of those plays consistent with $\left(\sigma_{E}, \sigma_{A}\right)$ is in bijection with the set of infinite paths in the Markov chain $\mathcal{M}_{\sigma_{E}, \sigma_{A}}^{\mathcal{G}}$. Hence, the associated probability measure $P_{\sigma_{E}, \sigma_{A}}^{\mathcal{G}}$ can be used as a probability measure for measurable subsets of Plays $\sigma_{\sigma_{E}, \sigma_{A}}^{\mathcal{G}}$.

When $\mathcal{G}$ is understood, we omit it and simply write $P_{\sigma_{E}, \sigma_{A}}$ and Plays $\sigma_{\sigma_{E}, \sigma_{A}}$

A winning condition is a subset ${ }^{3} \Omega \subseteq V^{\omega}$ and a (two-player perfect-information) stochastic game is a pair $\mathbb{G}=(\mathcal{G}, \Omega)$.

A strategy $\sigma_{E}$ for Éloïse is surely winning if Plays ${ }_{\sigma_{E}, \sigma_{A}} \subseteq \Omega$ for every strategy $\sigma_{A}$ of Abélard; it is almost-surely winning if $P_{\sigma_{E}, \sigma_{A}}(\Omega)=1$ for every strategy $\sigma_{A}$ of Abélard. Similar notions for Abélard are defined dually. Éloïse surely (resp. almost-surely) wins if she has a surely (resp. almost-surely) winning strategy.

A reachability game is a stochastic game whose winning condition is of the form $V^{*} F V^{\omega}$ for some subset $F \subseteq V$, i.e. winning plays are those that eventually visit a vertex in $F$. A Büchi game is a stochastic game whose winning condition is of the form $\bigcap_{i \geq 0} V^{i} V^{*} F V^{\omega}$ for some subset $F \subseteq V$, i.e. winning plays are those that infinitely often visit a vertex in $F$. Finally, a co-Büchi game is stochastic game whose winning condition is of the form $V^{*}(V \backslash F)^{\omega}$ for some subset $F \subseteq V$, i.e. winning plays are those that finitely often visit a vertex in $F$. When it is clear from the context, we write $\mathbb{G}=(\mathcal{G}, F)$ (i.e. write $F$ instead of $\Omega$ ) for the reachability (resp. Büchi, co-Büchi) game relying on $F$.

A positional strategy $\sigma$ is a strategy that does not require any memory, i.e. such that for any two histories of the form $\lambda \cdot v$ and $\lambda^{\prime} \cdot v$, one has $\sigma(\lambda \cdot v)=\sigma\left(\lambda^{\prime} \cdot v\right)$. Positional strategies only depend on the current vertex, and for convenience they are written as functions from $V$ into $V$.

A game is deterministic whenever $V_{R}=\emptyset$. It is well-known (see e.g. [33]) that positional strategies suffice to surely win in deterministic games with a parity winning condition, which we do not define but captures the reachability, Büchi, and co-Büchi winning conditions that we are interested in.

Theorem 2.1 (Positional Determinacy [33]). Let $\mathbb{G}$ be a deterministic parity game. Then, either Éloïse or Abélard has a positional surely winning strategy.

\footnotetext{
${ }^{2}$ We only consider pure strategies, as these are sufficient for our purpose. However, our main results on positionality (Theorems 3.1 and 3.2) remain true for randomised strategies as later discussed in Remark 3.4.

${ }^{3}$ Formally, one needs to require that $\Omega$ is measurable for the probability measure $P_{\sigma_{E}, \sigma_{A}}$, which is always trivially true in this paper.
} 
For stochastic games, the following result is well-known (see e.g. [21] for a slightly more general result).

THEOREM 2.2. Let $\mathbb{G}$ be a stochastic parity game played on a finite arena. If Éloïse almost-surely wins then she has an a positional almost-surely winning strategy.

Note that dropping the assumption that the arena is finite substantially changes the situation. Indeed, for infinite arenas, even with a reachability condition and assuming finite out-degree, almostsurely winning strategies for Éloïse may require infinite memory [23, Proposition 5.7]. However, imposing a natural structural restriction on the (possibly infinite) arena, namely to be chronological, yields a result like Theorem 2.2 for Büchi games, see Theorem 3.1.

\subsection{Two-Player Imperfect-Information Stochastic Büchi Games}

We now introduce a subclass of the usual games with imperfect information which is essentially a stochastic version of the model in [11]. Our model of imperfect-information games is quite restrictive compared to general models developed in [4, 9, 10,22], as in our setting Abélard is perfectly informed. However, it turns out to be expressive enough to be used as a central tool to check emptiness for alternating Büchi tree automata with qualitative semantics.

A stochastic arena of imperfect information is a tuple $\mathcal{G}=\left(V, A, T, \sim, v_{\text {in }}\right)$ where $V$ is a finite set of vertices, $v_{\text {in }} \in V$ is an initial vertex, $A$ is the finite alphabet of Éloïse's actions, $T \subseteq$ $V \times A \times \mathcal{D}(V)$ is a stochastic transition relation and $\sim$ is an equivalence relation over $V$ that denotes the observational capabilities of Éloïse and therefore imposes restrictions on legitimate strategies for her (see further). We additionally require that for all $(v, a) \in V \times A$ there is at least one $\delta \in \mathcal{D}(V)$ such that $(v, a, \delta) \in T$.

A play starts from the initial vertex $v_{i n}$ and proceeds as follows: Éloïse plays an action $a_{0} \in A$, then Abélard resolves the non-determinism by choosing a distribution $\delta_{0}$ such that $\left(v_{i n}, a_{0}, \delta_{0}\right) \in T$ and finally a new vertex is randomly chosen according to $\delta_{0}$. Then, Éloïse plays a new action, Abélard resolves the non-determinism and a new vertex is randomly chosen, and so on forever. Hence, a play is an infinite word $v_{\text {in }} a_{0} \delta_{0} v_{1} a_{1} \delta_{1} v_{2} \cdots \in(V \cdot A \cdot \mathcal{D}(V))^{\omega}$. A history is a prefix of a play ending in a vertex in $V$.

An imperfect-information stochastic Büchi game is a pair $\mathbb{G}=(\mathcal{G}, F)$ where $\mathcal{G}$ is a stochastic arena of imperfect information with a subset of states $F \subset V$ used to define the Büchi winning condition as follows: a play $\lambda=v_{0} a_{0} \delta_{0} v_{1} a_{1} \delta_{1} v_{2} \cdots$ in $\mathbb{G}$ is won by Éloïse if, and only if, the set $\left\{i \geq 0 \mid v_{i} \in F\right\}$ is infinite, i.e. winning plays are those that infinitely often visit a vertex in $F$.

The imperfect-information of the game is modelled by the equivalence relation $\sim$ that conveys which vertices Éloïse cannot distinguish, namely those that are $\sim$-equivalent. We will write $V_{/ \sim}$ for the set of equivalence classes of $\sim$ in $V$, and for every $v \in V$, we will write $[v]_{\sim}$ for its $\sim$-equivalence class.

Relation plays a crucial role when defining strategies for Éloïse. Intuitively, Éloïse should not play differently in two indistinguishable plays, where the indistinguishability of Éloïse is based on perfect recall [14]: Éloïse cannot distinguish two histories $v_{i n} a_{0} \delta_{0} v_{1} a_{1} \delta_{1} \cdots v_{\ell}$ and $v_{i n}^{\prime} a_{0}^{\prime} \delta_{0}^{\prime} v_{1}^{\prime} a_{1}^{\prime} \delta_{1}^{\prime} \cdots v_{\ell}^{\prime}$ whenever $v_{i} \sim v_{i}^{\prime}$ for all $i \leq \ell$ and $a_{i}=a_{i}^{\prime}$ for all $i<\ell$. Note that in particular, Éloïse does not observe Abélard's choices for the distributions along a play. Hence, a (pure $\left.{ }^{4}\right)$ strategy for Éloïse is a function $\sigma_{E}:\left(V_{/ \sim} \cdot A\right)^{*} \cdot\left(V_{/ \sim}\right) \rightarrow A$ assigning an action to every set of indistinguishable histories. Élö̈se respects a strategy $\sigma_{E}$ during a play $\lambda=v_{\text {in }} a_{0} \delta_{0} v_{1} a_{1} \delta_{1} \cdots$ if $a_{i+1}=\sigma_{E}\left(\left[v_{i n}\right]_{\sim} a_{0}\left[v_{1}\right]_{\sim} \cdots\left[v_{i}\right]_{\sim}\right)$, for all $i \geq 0$.

\footnotetext{
${ }^{4}$ Again, as for perfect information games, we do not consider randomised strategies as pure strategies are the right model for our purpose.
} 
A strategy for Abélard is defined as a function $\sigma_{A}:(V \cdot A \cdot \mathcal{D}(V))^{*}(V \cdot A) \rightarrow \mathcal{D}(V)$ such that $\left(v, a, \sigma_{A}(\lambda \cdot v \cdot a)\right) \in T$ for every $\lambda \in(V \cdot A \cdot \mathcal{D}(V))^{*}$. Abélard respects a strategy $\sigma_{A}$ during a play $\lambda=v_{\text {in }} a_{0} \delta_{0} v_{1} a_{1} \delta_{1} \cdots$ if $\delta_{i}=\sigma_{A}\left(v_{i n} a_{0} \delta_{0} v_{1} a_{1} \delta_{1} \cdots v_{i} a_{i}\right)$, for all $i \geq 0$.

Exactly as in the perfect-information setting, one associates with a pair of strategies $\left(\sigma_{E}, \sigma_{A}\right)$ the set Plays $\mathcal{G}_{\sigma_{E}, \sigma_{A}}^{\mathcal{G}}$ of those plays where Éloïse (resp. Abélard) respects $\sigma_{E}$ (resp. $\left.\sigma_{A}\right)$, and equip it with a probability measure.

Finally, a strategy $\sigma_{E}$ for Éloïse is almost-surely winning if, against any strategy $\sigma_{A}$ for Abélard, the set of winning plays for Éloïse has measure 1 for the probability measure on Plays $\sigma_{\sigma_{E}, \sigma_{A}}$.

Remark 2.3. It is important to note that Éloïse may not observe whether a vertex belongs to $F$ as we do not require that $v \sim v^{\prime} \Rightarrow\left(v \in F \Leftrightarrow v^{\prime} \in F\right)$. In particular, this has to be taken into account when eventually solving the game.

The following decidability result will be crucial in Section 3.2.

Theorem $2.4([9,10])$. Let $\mathbb{G}$ be an imperfect-information stochastic Büchi game. One can decide in exponential time whether Élö̈se has an almost-surely winning strategy in $\mathbb{G}$.

\subsection{Probabilistic Automata on Finite Words}

Probabilistic automata on finite words generalize non-deterministic automata by letting the transition function map a state and a letter to a distribution over states [28]. The reference book for early developments on probabilistic automata is due to Paz [26].

A probabilistic word automaton is a tuple $\mathcal{A}=\left(Q, q_{i n}, \delta\right)$, where $Q$ is the finite set of states, $q_{\text {in }}$ is the initial state, and $\delta: Q \times \Sigma \rightarrow \mathcal{D}(Q)$ is the transition function. We say that a probabilistic automaton is simple when the distribution $\delta(q, a)$ is always of the form $\frac{1}{2} q_{1}+\frac{1}{2} q_{2}$ (possibly with $\left.q_{1}=q_{2}\right)$.

Intuitively, a finite word $u=u_{1} \ldots u_{k} \in \Sigma^{*}$ induces a set of runs of $\mathcal{A}$ each of which comes with a probability of being realised; if one fixes a set of final states, the acceptance probability of $u$ by $\mathcal{A}$ is the mere sum of the probabilities of those runs of $\mathcal{A}$ over $u$ that end in a final state. To formally define acceptance probability (and extend it further to richer settings) we associate with $\mathcal{A}$ and $u$ a Markov chain $\mathcal{M}_{\mathcal{A}}^{u}$ as follows.

The Markov chain $\mathcal{M}_{\mathcal{A}}^{u}$ has the (finite) set of states $Q \times\{0, \ldots, k\}$, the initial state $\left(q_{\text {in }}, 0\right)$, and the probability transition function $T_{\mathcal{A}}^{u}$ defined for every $(p, i) \in Q \times\{0, \ldots, k-1\}$ (we do not define it for states of the form $(p, k)$ that will be useless) by

$$
T_{\mathcal{A}}^{u}((p, i))=\sum_{q \in Q} \delta\left(p, u_{i}\right)(q) \cdot(q, i+1)
$$

Call a finite path of length $k+1$ of $\mathcal{M}_{\mathcal{A}}^{u}$ a run of $\mathcal{A}$ on $u$ and let $P_{\mathcal{A}}^{u}$ be the probability measure on runs induced by $\mathcal{M}_{\mathcal{A}}^{u}$. Given a subset of (final) states $F \subseteq Q$, call Last $(F)$ the set of runs whose (first coordinate of the) last state is in $F$. We then define the acceptance probability of $\mathcal{A}$ over $u$ as $P_{\mathcal{A}}^{u}(\operatorname{Last}(F))$.

A classic decision problem for probabilistic word automata is the value 1 problem.

$$
\begin{aligned}
\text { INPUT: } & \text { A probabilistic word automaton } \mathcal{A} \text { and a subset } F \subseteq Q \\
\text { QUESTION: } & \forall \varepsilon>0, \exists u \in \Sigma^{*}, P_{\mathcal{A}}^{u}(\operatorname{Last}(F)) \geq 1-\varepsilon \text { ? }
\end{aligned}
$$

Informally, the value 1 problem asks for the existence of words with acceptance probabilities that are arbitrarily close to 1 . In this case, we say that $\mathcal{A}$ has value 1 . The undecidability of the value 1 problem for simple probabilistic automata was first established in [20] (see also [16] and [15] for a simple proof). 
THeORem 2.5 ([20]). The value 1 problem for simple probabilistic word automata is undecidable.

\subsection{Probabilistic Automata on Infinite Words}

Baier, Größer, and Bertrand conducted an in-depth study of probabilistic automata over infinite words [1]. To define the semantics of a probabilistic word automaton $\mathcal{A}=\left(Q, q_{i n}, \delta\right)$ over an infinite word $w=w_{1} w_{2} \cdots$, we proceed as for finite words and construct a Markov chain $\mathcal{M}_{\mathcal{A}}^{w}$ whose set of states is $Q \times \mathbb{N}$. The initial state is again $\left(q_{i n}, 0\right)$, and the probability transition function $T_{\mathcal{A}}^{w}$ is still defined by

$$
T_{\mathcal{A}}^{w}((p, i))=\sum_{q \in Q} \delta\left(p, w_{i}\right)(q) \cdot(q, i+1)
$$

A run of $\mathcal{A}$ on $w$ is now an infinite path in $\mathcal{M}_{\mathcal{A}}^{w}$ and the Markov chain yields a probability measure $P_{\mathcal{A}}^{w}$ on runs.

For probabilistic automata on infinite words we mostly focus on the co-Büchi acceptance condition that is defined as follows. Given a subset of states $F \subseteq Q$, we let co-Büchi $(F)=(Q \times \mathbb{N})^{*}((Q \backslash$ $F) \times \mathbb{N})^{\omega}$ be the (measurable) set of runs that visit $F$ only finitely often, and, when this set of runs has measure 1 , we say that $w$ is almost-surely accepted by $\mathcal{A}$ for the co-Büchi condition $F$, written $w \in L_{\operatorname{co}-\operatorname{Büchi}(F)}^{=1}(\mathcal{A})$. Formally,

$$
L_{\text {co-Büchi }(F)}^{=1}(\mathcal{A})=\left\{w \in \Sigma^{\omega}: P_{\mathcal{A}}^{w}(\operatorname{co}-\operatorname{Büchi}(F))=1\right\} .
$$

Example 2.6. Let $\Sigma$ be an alphabet and $\sharp \notin \sum$ be a fresh symbol. Let $C$ be the simple probabilistic co-Büchi automaton with set $\left\{p_{1}, p_{2}\right\}$ of states, initial state $p_{1}$, and transition function given by:

- $\delta\left(p_{1}, a\right)=p_{1}$ for any $a \in \Sigma \backslash\{\sharp\}$;

- $\delta\left(p_{1}, \sharp\right)=\frac{1}{2} p_{1}+\frac{1}{2} p_{2}$; and

- $\delta\left(p_{2}, a\right)=p_{2}$ for any $a \in \Sigma \cup\{\sharp\}$.

As $p_{2}$ is absorbing and as moving from $p_{1}$ to $p_{2}$ may only happen when reading $\sharp$, the language $L_{\text {co-Büchi }\left(\left\{p_{1}\right\}\right)}^{=1}(C)$ consists of those infinite words over $\Sigma \cup\{\sharp\}$ that contain infinitely many occurrences of $\sharp$. Note that we will later use this example as a gadget in the proof of Proposition 4.1

The emptiness problem for probabilistic co-Büchi word automata with almost-sure semantics is the following decision problem:

$$
\begin{array}{rl|}
\text { INPUT: } & \text { A probabilistic word automaton } \mathcal{A} \text { and a set } F \subseteq Q \\
\text { QUESTION: } & \text { Is } L_{\text {co-Büchi }(F)}^{=1}(\mathcal{A})=\emptyset \text { ? }
\end{array}
$$

It was shown in [1] that this problem is undecidable.

Proposition 2.7 ([1]). The emptiness problem for probabilistic co-Büchi word automata with almost-sure semantics is undecidable.

The proof in [1] is obtained by reducing the universality problem for simple probabilistic Büchi word automata with the positive semantics: Indeed, automata in this class (we refer to [1] for definitions) can be effectively complemented into probabilistic co-Büchi word automata with the almost-sure semantics, and whose universality problem is proved to be undecidable. As the complementation procedure does not preserve the property of being simple, we will later argue (see Proposition 4.1) that Proposition 2.7 still holds for simple probabilistic co-Büchi word automata with almost-sure semantics. 


\subsection{Universal Automata on Infinite Trees with Qualitative Semantics}

The qualitative semantics for tree automata was introduced by Carayol, Haddad, and Serre in [8] and was studied for non-deterministic [8], alternating [17], and probabilistic automata [8].

In this section, we define universal tree automata with qualitative semantics and then extend this concept to alternating tree automata with qualitative semantics in the next section.

A tree automaton is a tuple $\mathcal{A}=\left(Q, q_{\text {in }}, \Delta\right)$, where $Q$ is a finite set of states, $q_{\text {in }}$ is the initial state, and $\Delta \subseteq Q \times \Sigma \times Q \times Q$ is the transition relation. A run of $\mathcal{A}$ over a $\Sigma$-tree $t$ is a $Q$-tree $\rho:\{0,1\}^{*} \rightarrow Q$ such that $\rho(\varepsilon)=q_{\text {in }}$ and, for all $u \in\{0,1\}^{*}$, we have $(\rho(u), t(u), \rho(u 0), \rho(u 1)) \in \Delta$. We let Runs $\mathcal{A}(t)$ denote the set of runs of $\mathcal{A}$ over $t$.

A tree automaton $\mathcal{A}$ and a run $\rho$ induce a Markov chain $\mathcal{M}_{\mathcal{A}}^{\rho}$ as follows. The set of states is $Q \times\{0,1\}^{*}$, the initial state is $\left(q_{i n}, \varepsilon\right)$, and the probability transition function $T_{\mathcal{A}}^{\rho}$ is given by

$$
T_{\mathcal{A}}^{\rho}((\rho(u), u))=\frac{1}{2}(\rho(u 0), u 0)+\frac{1}{2}(\rho(u 1), u 1)
$$

yielding the probability measure $P_{\mathcal{A}}^{\rho}$ on branches of the run $\rho$.

Given a subset of states $F \subseteq Q$, we let co-Büchi $(F)=\left(Q \times\{0,1\}^{*}\right)^{*}\left((Q \backslash F) \times\{0,1\}^{*}\right)^{\omega}$ be the (measurable) set of infinite paths in $\mathcal{M}_{\mathcal{A}}^{\rho}$ that visit $F$ only finitely often, and we say that the run $\rho$ is qualitatively accepting for the co-Büchi condition $F$ if $P_{\mathcal{A}}^{\rho}(\operatorname{co}-\mathrm{Büchi}(F))=1$. Equivalently, a run $\rho$ is qualitatively accepting for the co-Büchi condition if and only if the set of branches in $\rho$ that contain finitely many nodes labelled by a state in $F$ has measure 1 for the classical coin-flipping measure $\mu$ on branches: $\mu$ is the unique complete probability measure such that $\mu\left(u \cdot\{0,1\}^{\omega}\right)=$ $2^{-|u|}$.

The universal semantics yields the following definition:

$$
L_{\text {Qual,co-Büchi }(F)}^{\forall}(\mathcal{A})=\left\{t \in \operatorname{Trees}(\Sigma): \forall \rho \in \operatorname{Runs}_{\mathcal{A}}(t), P_{\mathcal{A}}^{\rho}(\operatorname{co-Büchi}(F))=1\right\} .
$$

In words, a tree $t$ belongs to $L_{\text {Qual,co-Büchi }(F)}^{\forall}(\mathcal{A})$ if every run of $\mathcal{A}$ over $t$ is such that almost all its branches contain finitely many states in $F$.

The emptiness problem for universal co-Büchi tree automata with qualitative semantics is the following decision problem:

$$
\begin{aligned}
\text { INPUT: } & \text { A tree automaton } \mathcal{A} \text { and a set } F \subseteq Q \\
\text { QUESTION: } & \text { Is } L_{\text {Qual,co-Büchi }(F)}^{\forall}(\mathcal{A})=\emptyset ?
\end{aligned}
$$

We will prove in Theorem 4.2 that this problem is undecidable.

\subsection{Alternating Automata on Infinite Trees with Qualitative Semantics}

An alternating tree automaton is a tuple $\mathcal{A}=\left(Q, q_{i n}, Q_{E}, Q_{A}, \Delta\right)$, where $Q$ is the finite set of states, $q_{\text {in }}$ is the initial state, $\left(Q_{E}, Q_{A}\right)$ is a partition of $Q$ into Éloïse's and Abélard's states and $\Delta \subseteq Q \times \Sigma \times Q \times Q$ is the transition relation.

The input of such an automaton is a $\Sigma$-tree $t$ and acceptance is defined by means of the following two-player perfect-information stochastic game $\mathbb{G}_{\mathcal{A}, t}^{=1}$. Intuitively, a play in this game consists in moving a pebble along a branch of $t$ starting from the root: the pebble is attached to a state and in a node $u$ with state $q$, Éloïse (if $q \in Q_{E}$ ) or Abélard (if $q \in Q_{A}$ ) picks a transition $\left(q, t(u), q_{0}, q_{1}\right) \in \Delta$, and then Random chooses to move down the pebble either to node $u 0$ (and then updates the state to $q_{0}$ ) or to node $u 1$ (and then updates the state to $q_{1}$ ). 
Formally, let $G=\left(V_{E} \cup V_{A} \cup V_{R}, E\right)$ with $V_{E}=Q_{E} \times\{0,1\}^{*}, V_{A}=Q_{A} \times\{0,1\}^{*}$ and $V_{R}=$ $\left\{\left(q, u, q_{0}, q_{1}\right) \mid u \in\{0,1\}^{*}\right.$ and $\left.\left(q, t(u), q_{0}, q_{1}\right) \in \Delta\right\}$, and

$$
\begin{aligned}
E=\quad & \left\{\left((q, u),\left(q, u, q_{0}, q_{1}\right)\right) \mid\left(q, u, q_{0}, q_{1}\right) \in V_{R}\right\} \quad \cup \\
& \left\{\left(\left(q, u, q_{0}, q_{1}\right),\left(q_{x}, u \cdot x\right)\right) \mid x \in\{0,1\} \text { and }\left(q, u, q_{0}, q_{1}\right) \in V_{R}\right\}
\end{aligned}
$$

Then, we define $\mathcal{G}_{\mathcal{A}, t}^{=1}=\left(G, V_{E}, V_{A}, V_{R}, \delta,\left(q_{i n}, \varepsilon\right)\right)$ where $\delta\left(\left(q, u, q_{0}, q_{1}\right)\right)=\frac{1}{2}\left(q_{0}, u 0\right)+\frac{1}{2}\left(q_{1}, u 1\right)$.

Given a subset of states $F \subseteq Q$, we say that $t$ is qualitatively accepted by $\mathcal{A}$ for the Büchi (resp. co-Büchi) condition $F$ if Éloïse has an almost-surely winning strategy in the Büchi (resp. co-Büchi) game $\mathbb{G}_{\mathcal{A}, t}^{=1}=\left(\mathcal{G}_{\mathcal{A}, t}^{=1}, F \times\{0,1\}^{*}\right)$.

For an alternating tree automaton $\mathcal{A}$ and a subset of states $F$, we denote by $L_{\text {Qual,Büchi }(F)}^{\text {Alt }}(\mathcal{A})$ (resp. $\left.L_{\text {Qual,co-Büchi }(F)}^{\text {Alt }}(\mathcal{A})\right)$ the set of trees qualitatively accepted by $\mathcal{A}$ for the Büchi (resp. co-Büchi) condition $F$.

Remark 2.8. Any positional strategy for Éloïse in $\mathbb{G}_{\mathcal{A}, t}^{=1}$ can be described as a function $\sigma: Q_{E} \times$ $\{0,1\}^{*} \rightarrow Q \times Q$ that satisfies the following property: $\forall u \in\{0,1\}^{*}$, if $\sigma(q, u)=\left(q_{0}, q_{1}\right)$ then $\left(q, t(u), q_{0}, q_{1}\right) \in \Delta$. Equivalently, in a curried form, $\sigma$ is a map $\{0,1\}^{*} \rightarrow\left(Q_{E} \rightarrow Q \times Q\right)$. Hence, if one lets $\mathcal{T}$ be the set of functions from $Q_{E}$ into $Q \times Q$, Éloïse's positional strategies are in bijection with $\mathcal{T}$-labelled binary trees.

It is easily seen that universal tree automata with qualitative semantics are subsumed by alternating tree automata with qualitative semantics. Indeed we have the following classical result (that we state here only for co-Büchi acceptance condition but that works similarly for any other acceptance condition).

Proposition 2.9. Let $\mathcal{A}=\left(Q, q_{\text {in }}, \Delta\right)$ be a tree automaton and let $F \subseteq Q$. Consider the alternating tree automaton $\mathcal{B}=\left(Q, q_{\text {in }}, \emptyset, Q, \Delta\right)$, meaning that all states of $\mathcal{A}$ are interpreted as Abélard's. Then the following holds.

$$
L_{\text {Qual,co-Büchi(F) }}^{\forall}(\mathcal{A})=L_{\text {Qual,co-Büchi(F) }}^{\text {Alt }}
$$

Proof. For a fixed tree $t$, runs of $\mathcal{A}$ over $t$ are in bijection with strategies of Abélard in the co-Büchi game $\mathbb{G}_{\mathcal{B}, t}^{=1}$ (where Éloïse is making no choice), and moreover a run is qualitatively accepting for $\mathcal{A}$ if and only if Élö̈se almost-surely wins in $\mathbb{G}_{\mathcal{B}, t}^{=1}$ when Abélard uses the corresponding strategy. Hence, all runs of $\mathcal{A}$ over $t$ are qualitatively accepting if and only if Éloïse almostsurely wins against every strategy of Abélard in $\mathbb{G}_{\mathcal{B}, t}^{=1}$, which means that $L_{\text {Qual,co-Büchi }(F)}^{\forall}(\mathcal{A})=$ $L_{\text {Qual,co-Büchi }(F)}^{\text {Alt }}(\mathcal{B})$.

The emptiness problem for alternating Büchi tree automata with qualitative semantics is the following decision problem:

$$
\begin{aligned}
\text { INPUT: } & \text { An alternating tree automaton } \mathcal{A} \text { and a set } F \subseteq Q \\
\text { QUESTION: } & \text { Is } L_{\mathrm{Qual}, \mathrm{Büchi}(F)}^{\text {Alt }}(\mathcal{A})=\emptyset ?
\end{aligned}
$$

We will prove in Theorem 3.7 that this problem is decidable in exponential time.

Remark 2.10. The emptiness problem can be similarly defined for alternating co-Büchi tree automata with qualitative semantics. However, this problem is undecidable as a corollary of Proposition 2.9 together with the forthcoming Theorem 4.2, proving the undecidability of the emptiness problem for universal co-Büchi tree automata with qualitative semantics. 


\subsection{Probabilistic Automata on Infinite Trees with Qualitative Semantics}

Probabilistic tree automata with qualitative semantics were defined in [8] with the intention of lifting the definition of probabilistic automata on infinite words to the case of infinite trees. In particular, an input tree induces a probability distribution over runs and acceptance is defined by requiring that almost all runs should be accepting. Mixed with the qualitative co-Büchi semantics, this means that a tree is accepted if almost all runs have almost all their branches containing finitely many states from $F$. Contrary to the authors of [8] who define a probability measure on runs, we follow another approach (still yielding an equivalent notion [8, Proposition 45]) based on Markov chains.

A probabilistic tree automaton is a tuple $\mathcal{A}=\left(Q, q_{i n}, \delta\right)$, where $Q$ is the finite set of states, $q_{\text {in }}$ is the initial state, and $\delta: Q \times \Sigma \rightarrow \mathcal{D}(Q \times Q)$ is the transition function.

A probabilistic tree automaton $\mathcal{A}$ and a tree $t$ induce a Markov chain $\mathcal{M}_{\mathcal{A}}^{t}$ as follows. The set of states is $Q \times\{0,1\}^{*}$, the initial state is $\left(q_{i n}, \varepsilon\right)$, and the probability transition function $T_{\mathcal{A}}^{t}$ is given by (where $\cdot$ distributes over + )

$$
T_{\mathcal{A}}^{t}((q, u))=\sum_{q_{0}, q_{1} \in Q} \delta(q, t(u))\left(q_{0}, q_{1}\right) \cdot\left(\frac{1}{2} \cdot\left(q_{0}, u 0\right)+\frac{1}{2} \cdot\left(q_{1}, u 1\right)\right),
$$

Given a subset of states $F \subseteq Q$, we again let co-Büchi $(F)=\left(Q \times\{0,1\}^{*}\right)^{*}\left((Q \backslash F) \times\{0,1\}^{*}\right)^{\omega}$ be the (measurable) set of infinite paths in $\mathcal{M}_{\mathcal{A}}^{t}$ that visit $F$ only finitely often. Then the probability measure $P_{\mathcal{A}}^{t}$ induced by $\mathcal{M}_{\mathcal{A}}^{t}$ yields the following definition of the set of trees almost-surely qualitatively accepted by $\mathcal{A}$ :

$$
L_{\text {Qual,co-Büchi }(F)}^{\forall^{=1}}(\mathcal{A})=\left\{t \in \operatorname{Trees}(\Sigma): P_{\mathcal{A}}^{t}(\operatorname{co-Büchi}(F))=1\right\} .
$$

We now turn to our main decidability result about emptiness of alternating Büchi tree automata with qualitative semantics.

\section{DECIDABILITY OF THE EMPTINESS PROBLEM FOR ALTERNATING BÜCHI TREE AUTOMATA WITH QUALITATIVE SEMANTICS}

In this section, we prove Theorem 3.7 that states the decidability of the emptiness problem for alternating Büchi tree automata with qualitative semantics, which contrasts with the forthcoming result that the emptiness problem for universal co-Büchi tree automata with qualitative semantics is undecidable (Theorem 4.2 of Section 4).

Our approach for checking emptiness of an alternating Büchi tree automaton $\mathcal{A}$ with qualitative semantics relies on a two-player imperfect-information stochastic finite Büchi game. In this game, Éloïse almost-surely wins if, and only if, the language accepted by $\mathcal{A}$ is non-empty. As for this class of games, one can decide whether Éloïse has an almost-surely winning strategy, the announced decidability result follows.

We establish in Section 3.1 a preliminary general positionality result to be used in Section 3.2 for proving the equivalence between Eloïse almost-surely winning in the game and $\mathcal{A}$ accepting some tree.

\subsection{A Positionality Result for Chronological Games}

For the rest of this section, we fix a stochastic arena $\mathcal{G}=\left(G, V_{E}, V_{A}, V_{R}, \delta, v_{\text {in }}\right)$ with $G=(V, E)$. Moreover, we assume that the game is chronological in the sense that there exists a function rank : $V_{E} \cup V_{A} \cup V_{R} \rightarrow \mathbb{N}$ such that $\operatorname{rank}^{-1}(0)=\left\{v_{\text {in }}\right\}$ and for $\left(v, v^{\prime}\right) \in E, \operatorname{rank}\left(v^{\prime}\right)=\operatorname{rank}(v)+1$. Note that the arena $\mathcal{G}_{\mathcal{A}, t}^{=1}$ used in Section 2.6 to define acceptance of a tree $t$ by an alternating tree 
automaton with qualitative semantics $\mathcal{A}$ is chronological. Note also that a chronological arena with finite out-degree has a countable set of vertices.

THEOREM 3.1. In a two-player perfect-information stochastic Büchi game played on a chronological arena with finite out-degree, Elö̈se has an almost-surely winning strategy if, and only if, she has a positional almost-surely winning strategy.

Actually, the core difficulty lies in proving Theorem 3.1 for the simple case of reachability games.

THEOREM 3.2. In a two-player perfect-information stochastic reachability game played on a chronological arena with finite out-degree, Eloïse has an almost-surely winning strategy if, and only if, she has a positional almost-surely winning strategy.

Proof. The direction from right to left is immediate. For the other direction, the key steps are the following. First, we establish (Lemma 3.3) that if Éloïse can ensure to reach $F$ with probability 1 from some initial vertex, then there exists a bound $k$ such that she can ensure to reach $F$ with probability at least half within $k$ steps. Second, we exploit Lemma 3.3 to "slice" the arena into infinitely many disjoint finite arenas: in each slice Éloïse plays to reach $F$ with probability at least half. Since each slice forms a finite sub-arena, optimal positional strategies always exist. Finally, the strategy that plays in turns the latter positional strategies ensures to almost-surely reach $F$ in the long run.

Let $\mathbb{G}=(\mathcal{G}, F)$ be a two-player perfect-information stochastic reachability game played on a chronological arena with finite out-degree. In the following, a strategy in $\mathbb{G}$ from a vertex $v$ is a strategy in the game obtained from $\mathbb{G}$ by changing the initial vertex of the arena $\mathcal{G}$ to $v$.

The following lemma allows us to decompose the infinite arena $\mathcal{G}$ into infinitely many finite arenas.

LEMmA 3.3. Let $\sigma_{E}$ be an almost-surely winning strategy for Éloïse in $\mathbb{G}$ from some vertex v. Then, there exists an integer $k$ such that for any strategy $\sigma_{A}$ of Abélard, we have

$$
\operatorname{Pr}_{\sigma_{E}, \sigma_{A}}\left(V^{\leq k} F V^{\omega}\right) \geq \frac{1}{2} \text {. }
$$

Proof of Lemma 3.3. Toward a contradiction, assume that such a $k$ does not exist. Hence, for each $k$ there exists a strategy $\sigma_{A, k}$ such that $\operatorname{Pr}_{\sigma_{E}, \sigma_{A, k}}\left(V^{\leq k} F V^{\omega}\right)<\frac{1}{2}$.

Without loss of generality, we can assume that $\sigma_{A, k}$ is positional. Indeed, one can pick for $\sigma_{A, k}$ a strategy for Abélard that minimises the probability of winning for Éloïse in the reachabililty game obtained by restricting $\mathbb{G}$ to vertices of rank at most $k$. This game has a finite arena since $\mathbb{G}$ has finite out-degree, and by e.g. [21] such a strategy for Abélard can be chosen positional.

From the sequence of strategies $\left(\sigma_{A, k}\right)_{k \geq 0}$, we now extract a strategy $\sigma_{A, \infty}$ (designed to contradict the assumption that Éloïse has an almost-surely winning strategy) that for every $k \geq 0$, agrees with infinitely many $\sigma_{A, h}$ on its first $k$ moves. Since $\mathbb{G}$ has countably many vertices, fix an (arbitrary) enumeration $v_{1}, v_{2}, \cdots$ of the vertices in $V$.

We define $\sigma_{A, \infty}$ step-wise inductively on $i$ at step $i, \sigma_{A, \infty}$ is defined on $v_{1}, \cdots, v_{i}$ and on these vertices agrees with all those strategies $\sigma_{A, h}$ with $h \in I_{i}$ where the sequence $I_{0} \supseteq I_{1} \supseteq I_{2} \supseteq I_{3} \supseteq \cdots$ is also defined inductively on $i$ and is such that each $I_{i}$ is infinite.

We let $I_{0}=\mathbb{N}$ be the set of all positive integers.

For $I_{i}$ where $i \geq 1$, consider the values of $\sigma_{A, h}\left(v_{i}\right)$ for all $h \in I_{i-1}$. Because $G$ has finite out-degree, there is some $v$ such that $\sigma_{A, h}\left(v_{i}\right)=v$, for infinitely many $h \in I_{i-1}$. We define $\sigma_{A, \infty}\left(v_{i}\right)=v$ and we let $I_{i}=\left\{h \in I_{i-1} \mid \sigma_{A, h}\left(v_{i}\right)=v\right\}$; note that $I_{i}$ is infinite.

Now, for $k \geq 0$, it is easy to see that by choosing $i$ big enough so that all vertices of rank at most $k$ belong to $\left\{v_{1}, \ldots, v_{i}\right\}$, strategy $\sigma_{A, \infty}$ agrees on its $k$ first moves with the infinitely many $\sigma_{A, h}$ where $h \in I_{i}$. 
As a consequence, for every $k$ there is some $h \geq k$ such that

$$
\operatorname{Pr}_{\sigma_{E}, \sigma_{A, \infty}}\left(V^{\leq k} F V^{\omega}\right)=\operatorname{Pr}_{\sigma_{E}, \sigma_{A, h}}\left(V^{\leq k} F V^{\omega}\right) \leq \operatorname{Pr}_{\sigma_{E}, \sigma_{A, h}}\left(V^{\leq h} F V^{\omega}\right)<\frac{1}{2}
$$

As $V^{*} F V^{\omega}=\bigcup_{k \geq 0} V^{\leq k} F V^{\omega}$ and as the sequence $\left(V^{\leq k} F V^{\omega}\right)_{k \geq 0}$ is increasing for set inclusion, one concludes that

$$
\operatorname{Pr}_{\sigma_{E}, \sigma_{A, \infty}}\left(V^{*} F V^{\omega}\right)=\lim _{k \rightarrow \infty} \operatorname{Pr}_{\sigma_{E}, \sigma_{A, \infty}}\left(V^{\leq k} F V^{\omega}\right) \leq \frac{1}{2}<1
$$

which leads to a contradiction with $\sigma_{E}$ being almost-surely winning, and concludes the proof of Lemma 3.3.

Keeping on with the proof of Theorem 3.2, assume that Éloïse has an almost-surely wining strategy $\sigma_{E}$ in $\mathbb{G}$. Without loss of generality, we can assume that she has an almost-surely winning strategy from everywhere, by restricting the arena to vertices reachable by an almost-surely winning strategy.

For $k<k^{\prime}$, we define the reachability game $\mathbb{G}_{\left[k, k^{\prime}\right]}$ induced by restricting the arena $\mathcal{G}=$ $\left(G, V_{E}, V_{A}, V_{R}, \delta, v_{i n}\right)$ to vertices of rank in $\left[k, k^{\prime}\right]$ where we add self-loops on vertices of rank $k^{\prime}$ to avoid having dead-end vertices. Since $G$ has finite out-degree, there are finitely many vertices of rank in $\left[k, k^{\prime}\right]$, hence $\mathbb{G}\left[k, k^{\prime}\right]$ is finite.

We define inductively an increasing sequence of ranks $\left(k_{i}\right)_{i \geq 1}$ together with a sequence of strategies $\left(\sigma_{E,\left[k_{i}, k_{i+1}[\right.}\right)_{i \geq 1}$ such that for all $i \geq 1, \sigma_{E,\left[k_{i}, k_{i+1}[\right.}$ is a positional strategy, defined on all vertices of rank in $\left[k_{i}, k_{i+1}\left[\right.\right.$, and such that from all vertices of rank $k_{i}$, for all strategies $\sigma_{A}$, we have

$$
\operatorname{Pr}_{\sigma_{E,\left[k_{i}, k_{i+1}[\right.}, \sigma_{A}}\left(V^{\leq \ell} F V^{\omega}\right) \geq \frac{1}{2},
$$

where $\ell=k_{i+1}-k_{i}$.

Assume the first $i$ ranks and strategies are defined. For each vertex of rank $k_{i}$, Lemma 3.3 gives the existence of some bound $k$; since there are finitely many such vertices, we can consider the maximum of those bounds that we call $\ell$, and we let $k_{i+1}=k_{i}+\ell$. By construction and Lemma 3.3, from all vertices of rank $k_{i}$, for all strategies $\sigma_{A}$, we have

$$
\operatorname{Pr}_{\sigma_{E}, \sigma_{A}}\left(V^{\leq \ell} F V^{\omega}\right) \geq \frac{1}{2},
$$

where $\ell=k_{i+1}-k_{i}$. In other words, Éloïse wins the reachability game $\mathbb{G}_{\left[k_{i}, k_{i+1}\right]}$ with probability at least half, so, relying on a generalisation ${ }^{5}$ of Theorem 2.2 (see e.g. $[21,23]$ ), there exists an

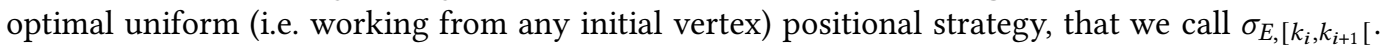
This concludes the inductive construction.

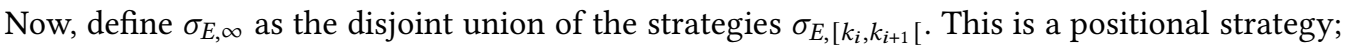
we argue that it is almost-surely winning. Assume, towards a contradiction, that this is not the case. Then, there exists $\varepsilon>0$ and a strategy $\sigma_{A}$ such that

$$
\operatorname{Pr}_{\sigma_{E, \infty}, \sigma_{A}}\left(V^{*} F V^{\omega}\right) \leq 1-\varepsilon .
$$

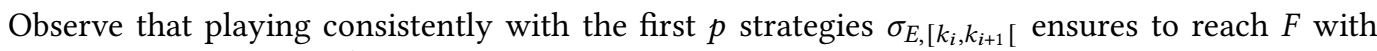
probability at least $1-\frac{1}{2^{p}}$. Since playing consistently with $\sigma_{E, \infty}$ implies playing consistently with the first $p$ strategies $\sigma_{E,\left[k_{i}, k_{i+1}[\right.}$, we reach a contradiction by considering $p$ large enough so that $\frac{1}{2^{p}}<\varepsilon$.

\footnotetext{
${ }^{5}$ More precisely, when playing a reachability game on a finite arena, Élö̈se always has an optimal positional strategy, where $\sigma_{E}$ being optimal means that inf $\sigma_{A} \operatorname{Pr}_{\sigma_{E}, \sigma_{A}}\left(V^{\leq \ell} F V^{\omega}\right)=\sup _{\sigma_{E}^{\prime}} \inf _{\sigma_{A}} \operatorname{Pr}_{\sigma_{E}, \sigma_{A}^{\prime}}\left(V^{\leq \ell} F V^{\omega}\right)$.
} 
Theorem 3.1 is an easy consequence of Theorem 3.2 thanks to a simple and neat reduction from [10, Remark 2.3] (also see [1, Lemma 8.3]). Roughly speaking, to turn a Büchi game into a reachability game equivalent with respect to almost-sure winning, one adds a unique final vertex and replaces every Büchi vertex by a fresh random vertex which either reaches the final vertex or proceeds in the game, each with probability half. Then, visiting infinitely many Büchi vertices ensures to almost-surely reach the final vertex, and conversely, reaching almost-surely the final vertex requires to almost-surely visit infinitely many Büchi vertices.

We make all this more formal.

Proof of Theorem 3.1. Recall that we denote by $\mathcal{G}=\left(G, V_{E}, V_{A}, V_{R}, \delta, v_{i n}\right)$, with $G=(V, E)$, the underlying arena of $\mathbb{G}$ and denote by $F \subseteq V$ the set of vertices defining the Büchi condition. We now build an arena $\mathcal{G}^{\prime}=\left(G^{\prime}, V_{E}^{\prime}, V_{A}^{\prime}, V_{R}^{\prime}, \delta^{\prime}, v_{i n}^{\prime}\right)$, with $G^{\prime}=\left(V^{\prime}, E^{\prime}\right)$, and a set $F^{\prime} \subseteq V^{\prime}$ of vertices such that Éloïse almost-surely wins in the Büchi game $\mathbb{G}=(G, F)$ if and only if she almost-surely wins in the reachability game $\mathbb{G}^{\prime}=\left(\mathcal{G}^{\prime}, F^{\prime}\right)$, and in addition, if she has a positional almost-surely winning strategy in one game, she has one in the other. This permits to deduce Theorem 3.1 from Theorem 3.2 .

We formally explain how to construct $\mathcal{G}^{\prime}$, taking care that it is chronological. The set of vertices $V^{\prime}$ consists of $V$ augmented with a countable set of vertices $\left\{f_{i} \mid i \geq 0\right\}$, and with extra random vertices $F_{R}=\left\{v_{s} \mid s \in F\right\}$, one per vertex in $F$. The vertex $f_{0}$ has a unique outgoing transition to $f_{1}$ and it can be reached only from vertices in $F_{R}$. For every $i \geq 1$, the vertex $f_{i}$ has a unique outgoing transition to $f_{i+1}$ and it can be reached only from $f_{i-1}$. From a vertex $v_{s} \in F_{R}$ there are two outgoing edges: one to $f_{0}$ and one to $s$ and both can be chosen with the same probability half, i.e. $\delta^{\prime}\left(v_{s}\right)=\frac{1}{2} f_{0}+\frac{1}{2} s$. Any edge in $G$ going from a vertex $v \in V$ to a vertex $s \in F$ is replaced by an edge from $v$ to $v_{s}$, and if $v \in V_{R}$ we let $\delta^{\prime}(v)(s)=0$ and $\delta^{\prime}(v)\left(v_{s}\right)=\delta(v)(s)$. All other edges are left untouched: for every $v \in V_{R}$ and $s \notin F \cup F_{R} \cup\left\{f_{i} \mid i \geq 0\right\}$, we let $\delta^{\prime}(v)(s)=\delta(v)(s)$. Finally we let $V_{E}^{\prime}=V_{E} \cup\left\{f_{i} \mid i \geq 0\right\}, V_{A}^{\prime}=V_{A}, V_{R}^{\prime}=V_{R} \cup F_{R}$ and $F^{\prime}=\left\{f_{0}\right\}$. Note that $\mathcal{G}^{\prime}$ is chronological by construction and $\mathcal{G}$ being chronological.

There is an obvious correspondence between strategies (of both Éloïse and Abélard) in $\mathbb{G}$ and strategies in $\mathbb{G}^{\prime}$, and it preserves positionality. Moreover, Éloïse almost-surely reaches the final state $f_{0}$ in $\mathbb{G}^{\prime}$ with strategy $\sigma_{E}^{\prime}$ if and only if she almost-surely visits infinitely often $F$ in $\mathbb{G}$ with the corresponding strategy $\sigma_{E}$. Indeed, if she almost-surely visits $F$ in $\mathbb{G}$ using $\sigma_{E}$, due to positive transition probability to $f_{0}$ from states in $F$, she almost-surely reaches $f_{0}$ in $\mathbb{G}^{\prime}$ using $\sigma_{E}^{\prime}$. Conversely, if against any strategy $\sigma_{E}$ of Éloïse in $\mathbb{G}$, Abélard has a strategy $\sigma_{A}$ that ensures that $F$ is visited finitely often with some positive probability $\varepsilon>0$, then in $\mathbb{G}^{\prime}$, when Éloïse and Abélard use the corresponding pair of strategies $\left(\sigma_{E}^{\prime}, \sigma_{A}^{\prime}\right)$, there is a positive probability $\varepsilon^{\prime}$ that $f_{0}$ is never reached, as the only way of reaching $f_{0}$ is by going through $F$; hence, in $\mathbb{G}^{\prime}$, against any strategy $\sigma_{E}^{\prime}$ of Éloïse, Abélard has a strategy $\sigma_{A}^{\prime}$ that avoids reaching $f_{0}$ with positive probability.

Remark 3.4. As already announced, in this paper we only considered pure (i.e. non-randomised) strategies. Hence, "Élö̈se has an almost-surely winning strategy" should be understood in both Theorem 3.2 and Theorem 3.1 as "Élö̈se has an almost-surely winning pure strategy". However, our proof directly carries over to the more general case of randomised strategies.

\subsection{Checking Emptiness}

Fix an alternating tree automaton $\mathcal{A}=\left(Q, q_{i n}, Q_{E}, Q_{A}, \Delta\right)$ and a subset $F \subseteq Q$ of final states. In order to check whether $L_{\text {Qual,Büchi }(F)}^{\text {Alt }}(\mathcal{A})=\emptyset$, we design an imperfect-information stochastic Büchi game $\mathbb{G}_{\mathcal{A}}^{\emptyset}$ in which Éloïse has an almost-surely winning strategy if and only if $L_{\text {Qual,Büchi }(F)}^{\text {Alt }}(\mathcal{A}) \neq \emptyset$. 
The equivalence is proved by applying the positionality result established in Theorem 3.1 to the acceptance game for $\mathcal{A}$.

In the game, Éloïse describes both a tree $t$ and a positional strategy $\sigma_{t}$ for her in the game $\mathbb{G}_{\mathcal{A}, t}^{=1}$. Following Remark 2.8, the positional strategy $\sigma_{t}$ is described as a $\mathcal{T}$-labelled tree, where $\mathcal{T}$ denotes the set of functions from $Q_{E}$ into $Q \times Q$. As the plays are of $\omega$-length, Éloïse actually does not fully describe $t$ and $\sigma_{t}$ but only a branch: this branch is chosen by Random while Abélard takes care of computing the sequence of states along it (either by updating an existential state according to $\sigma_{t}$ or, when the state is universal, by choosing an arbitrary valid transition of the automaton). In this game, Éloïse observes the directions, but not the actual control state of the automaton.

Remark 3.5. The fact that Éloïse does not observe the control state of the automaton is crucial here, as it avoids her to cheat when describing the input tree. Indeed, consider an alternating tree automaton whose initial state belongs to Abélard and from which there are two possible transitions: one that makes the automaton check that both subtrees only contain nodes labelled by $a$, and one that makes the automaton check that both subtrees only contain nodes labelled by $b$. Trivially, no tree is accepted by such an automaton. However, if one plays a modified version of the previous game where Éloïse observes the control state she can surely win in this game by producing a tree with all nodes labeled by $a$ (resp. by $b$ ) depending on the initial choice by Abélard.

Formally, we let $\mathcal{G}_{\mathcal{A}}^{\emptyset}=\left(V, A, T, \sim, v_{\text {in }}\right)$ where

- $V=(Q \times\{0,1\}) \cup\left\{\left(q_{\text {in }}, \varepsilon\right)\right\}$;

- $v_{\text {in }}=\left(q_{\text {in }}, \varepsilon\right)$;

- $A \subseteq \Sigma \times \mathcal{T}$ is the set $\left\{(a, \tau) \mid \forall q \in Q_{E},\left(q, a, q_{0}, q_{1}\right) \in \Delta\right.$ where $\left.\left(q_{0}, q_{1}\right)=\tau(q)\right\}$;

- $T=\left\{\left((q, i),(a, \tau), d_{q_{0}, q_{1}}\right) \mid q \in Q_{E}\right.$ and $\left.\tau(q)=\left(q_{0}, q_{1}\right)\right\} \cup$

$\left\{\left((q, i),(a, \tau), d_{q_{0}, q_{1}}\right) \mid q \in Q_{A}\right.$ and $\left.\left(q, a, q_{0}, q_{1}\right) \in \Delta\right\}$ where $d_{q_{0}, q_{1}}=\frac{1}{2}\left(q_{0}, 0\right)+\frac{1}{2}\left(q_{1}, 1\right)$; and

- $(q, i) \sim\left(q^{\prime}, i\right)$ for all $q, q^{\prime} \in Q$ and $i \in\{0,1\}$.

Finally we let $\mathbb{G}_{\mathcal{A}}^{\emptyset}=\left(\mathcal{G}_{\mathcal{A}}^{\emptyset}, F \times\{0,1\}\right)$.

The following theorem relates $\mathbb{G}_{\mathcal{A}}^{\emptyset}$ and $L_{\text {Qual,Büchi }(F)}^{\text {Alt }}(\mathcal{A})$.

THEOREM 3.6. Éloïse almost-surely wins in $\mathbb{G}_{\mathcal{A}}^{\emptyset}$ iff $L_{\text {Qual,Büchi }(F)}^{\text {Alt }}(\mathcal{A}) \neq \emptyset$.

Proof. Due to how $\sim$ is defined, a strategy for Éloïse in $\mathbb{G}_{\mathcal{A}}^{\emptyset}$ can also be viewed as a map $\sigma$ : $\{0,1\}^{*} \rightarrow A$. As $A \subseteq \Sigma \times \mathcal{T}$, one can see $\sigma$ as a pair $\left(t, \sigma_{t}\right)$ where $t$ is an infinite $\Sigma$-labelled binary tree, and $\sigma_{t}$ is a positional strategy for Éloïse in the acceptance game $\mathbb{G}_{\mathcal{A}, t}^{=1}$. Now, once such a strategy $\sigma$ is fixed, the set of plays in $\mathbb{G}_{\mathcal{A}}^{\emptyset}$ where Éloïse respects $\sigma$ is in one-to-one correspondence with the set of plays in $\mathbb{G}_{\mathcal{A}, t}^{=1}$ where she respects $\sigma_{t}$, and this correspondence preserves the property of being a winning play. Therefore, $\sigma=\left(t, \sigma_{t}\right)$ is almost-surely winning in $\mathbb{G}_{\mathcal{A}}^{\emptyset}$ iff $\sigma_{t}$ is an almostsurely winning positional strategy in $\mathbb{G}_{\mathcal{A}, t}^{=1}$ iff $t \in L_{\text {Qual,Büchi }(F)}^{\operatorname{Alt}}(\mathcal{A})$. The last equivalence holds because, thanks to Theorem 3.1, we can restrict our attention to positional strategies for Éloïse in the perfect-information game $\mathbb{G}_{\mathcal{A}, t}^{=1}$ which, we recall, is chronological and of course has finite out-degree. Finally, Éloïse has an almost-surely winning strategy in $\mathbb{G}_{\mathcal{A}}^{\emptyset}$ iff there exists some tree $t \in L_{\text {Qual,Büchi }(F)}^{\text {Alt }}(\mathcal{A})$.

Combining Theorem 3.6 with Theorem 2.4 directly implies decidability of the emptiness problem for alternating Büchi tree automata with qualitative semantics.

THEOREM 3.7. The emptiness problem for alternating Büchi tree automata with qualitative semantics is decidable in exponential time. 
Regarding lower bound, following the same ideas as in the undecidability proof in Theorem 4.2, one can reduce the emptiness problem for simple probabilistic Büchi automata with almost-sure semantics to the emptiness problem for universal ${ }^{6}$ Büchi tree automata with qualitative semantics.

THEOREM 3.8. The emptiness problem for universal Büchi tree automata with qualitative semantics is hard for ExpTime.

Proof. Similarly to what was done in Section 2.4 for the co-Büchi acceptance condition, we define a probabilistic Büchi automaton with almost-sure semantics on infinite words: for a probabilistic automaton $\mathcal{A}=\left(Q, q_{i n}, \delta\right)$ and a subset of states $F \subseteq Q$, we let $\operatorname{Büchi}(F)=\bigcap_{i \geq 0} Q^{i} Q^{*} F Q^{\omega}$ be the (measurable) set of runs that visit $F$ infinitely often. We then let:

$$
L_{\text {Büchi }(F)}^{=1}(\mathcal{A})=\left\{w \in \Sigma^{\omega}: P_{\mathcal{A}}^{w}(\operatorname{Büchi}(F))=1\right\} .
$$

The emptiness problem for probabilistic Büchi word automata with almost-sure semantics is the following decision problem:

$$
\begin{aligned}
\text { INPUT: } & \text { A probabilistic word automaton } \mathcal{A} \text { and a set } F \subseteq Q \\
\text { QUESTION: } & \text { Is } L_{\text {Büchi }(F)}^{=1}(\mathcal{A})=\emptyset ?
\end{aligned}
$$

It is proved in [1] that this problem is complete for ExpTime. Moreover, this result still holds with the extra requirement that the automata are simple. Indeed, the lower bound in [1] is by reduction of the almost-sure repeated reachability for partial-observation Markov decision processes. This latter problem was shown to be ExpTime-complete by de Alfaro [13]. The hardness proof in [13], based on the concept of blindfold games as defined by Reif in his seminal paper [31], survives (with the same proof) if the branching in the partial-observation Markov decision process has at most two states. Consequently, hardness for ExpTime already holds for probabilistic automata whose distributions involved in the transition function have a support of at most two states. Finally, as observed in [1, Remark 8.9], emptiness is not affected by changing the probabilities in the distributions as long as the support is unchanged: therefore, one can always reduce to the case of simple automata.

Now, following exactly the same path as in Theorem 4.2 one proves that the emptiness problem for simple probabilistic Büchi automata with almost-sure semantics can be polynomially reduced to the emptiness problem for universal Büchi tree automata with qualitative semantics, which implies the announced lower-bound.

\section{UNDECIDABILITY OF THE EMPTINESS PROBLEM FOR UNIVERSAL CO-BÜCHI TREE AUTOMATA WITH QUALITATIVE SEMANTICS}

In this section we prove our main undecidability result on the emptiness problem for universal coBüchi tree automata with qualitative semantics, from which we will then derive the undecidability of $\mathrm{MSO}+\forall_{\text {path }}^{=1}$ in Section 5 . We prove this result by reduction from the emptiness problem for simple probabilistic co-Büchi word automata with almost-sure semantics. As already mentioned (Proposition 2.7) it was shown in [1] that this problem is undecidable for general probabilistic word automata, but in our reduction to probabilistic tree automata it will be crucial to work with simple ones. We thus start by giving a proof of this slightly stronger result.

Proposition 4.1. The emptiness problem for simple probabilistic co-Büchi word automata with almost-sure semantics is undecidable.

\footnotetext{
${ }^{6}$ Following Proposition 2.9, we call universal an alternating Büchi tree automata whose set of states belonging to Éloïse is empty.
} 
Proof. The proof is by reduction from the value 1 problem for simple probabilistic automata, which is undecidable (Theorem 2.5).

Let $\mathcal{A}=\left(Q, q_{\text {in }}, \delta\right)$ be a simple probabilistic word automaton over some alphabet $\Sigma$, and let $F \subseteq Q$. Let $\sharp \notin \Sigma$ be a fresh symbol and let $\mathcal{A}^{\prime}=\left(Q \cup\left\{q_{i n}^{\prime}\right\}, q_{i n}, \delta^{\prime}\right)$ be the simple probabilistic automaton over $\Sigma \cup\{\sharp\}$ obtained from $\mathcal{A}$ as follows:

- $q_{i n}^{\prime}$ is a new state with $\delta^{\prime}\left(q_{i n}^{\prime}, a\right)=\delta\left(q_{i n}, a\right)$, for any letter $a \neq \sharp$, and $\delta^{\prime}\left(q_{i n}, \sharp\right)=q_{i n}^{\prime}$;

- $\delta^{\prime}(q, a)=\delta(q, a)$, for any state $q \in Q$ and any letter $a \neq \sharp$;

- $\delta^{\prime}(q, \sharp)=q_{\text {in }}$ if $q \in F$ and $\delta^{\prime}(q, \sharp)=q_{\text {in }}^{\prime}$ otherwise, for any state $q \in Q$.

We equip $\mathcal{A}^{\prime}$ with the co-Büchi condition $\left\{q_{\text {in }}^{\prime}\right\}$. Note that $\mathcal{A}^{\prime}$ is simple.

For a sequence of words $\left(u_{i}\right)_{i \geq 1}$ over $\Sigma$ we let $x_{i}$ be the acceptance probability of $\mathcal{A}$ over $u_{i}$, for every $i \geq 1$. Now consider an infinite word of the form $w=\sharp u_{1} \sharp u_{2} \sharp u_{3} \cdots$, and let $E_{i}$ be the event: “ $\mathcal{A}^{\prime}$ ends in $q_{i n}^{\prime}$ when it reads $u_{i} \sharp$ from $q_{i n}$ or $q_{i n}^{\prime}$ ". Each $E_{i}$ has probability $1-x_{i}$, and they are mutually independent. Also, $w$ is almost-surely accepted by $\mathcal{A}^{\prime}$ if and only if the probability that infinitely many of the events $E_{i}$ occur is zero. It is then a direct consequence of the Borel-Cantelli Lemma (and its converse) that $w$ is almost-surely accepted by $\mathcal{A}^{\prime}$ if and only if $\sum_{i=1}^{\infty} 1-x_{i}<\infty$.

It follows that $\mathcal{A}$ has value 1 if and only if $\mathcal{A}^{\prime}$ almost-surely accepts a word of the form $w=$ $\sharp u_{1} \sharp u_{2} \sharp u_{3} \cdots$. Indeed, if $\mathcal{A}$ has value 1 then there is a sequence of words $\left(u_{i}\right)_{i \geq 1}$ such that $x_{i} \geq 1-\frac{1}{i^{2}}$ and therefore such that $\sum_{i=1}^{\infty} 1-x_{i}<\infty$; conversely, if a sequence of words $\left(u_{i}\right)_{i \geq 1}$ is such that $\sum_{i=1}^{\infty} 1-x_{i}<\infty$, one must have $\lim _{x \rightarrow \infty} x_{i}=1$.

To conclude the proof it is sufficient to build a simple probabilistic co-Büchi word automaton $\mathcal{B}$ that almost-surely accepts only those words that are almost-surely accepted by $\mathcal{A}^{\prime}$, starting with a $\sharp$ and containing infinitely many $\sharp$.

Consider the automaton $C$ from Example 2.6 and recall that, when equipped with the acceptance condition co-Büchi $\left(\left\{p_{1}\right\}\right)$, it accepts those infinite words over $\Sigma \cup\{\sharp\}$ that contain infinitely many occurrences of $\sharp$.

Now, define $\mathcal{B}$ as the simple probabilistic automaton consisting of a fresh initial state $q_{\text {in }}^{\prime \prime}$ together with a copy of $\mathcal{A}^{\prime}$ and a copy of $C$. From $q_{i n}^{\prime \prime}$ the only possible action is to read a $\sharp$ and go either to the initial state of $\mathcal{B}$ with probability $\frac{1}{2}$ or to the initial state of $C$ with probability $\frac{1}{2}$.

Then it is immediate that $L_{\text {co-Büchi }\left(\left\{q_{i n}^{\prime}, p_{1}\right\}\right)}^{=1}(\mathcal{B})$ is empty if and only if $\mathcal{A}$ does not have value 1 .

Our main undecidability result of Theorem 4.2 contrasts with two decidability results, for probabilistic Büchi tree automata [8] and for alternating Büchi tree automata [17] (Theorem 3.7), both with qualitative semantics.

THeOREM 4.2. The emptiness problem for universal co-Büchi tree automata with qualitative semantics is undecidable.

To prove Theorem 4.2 we construct a reduction from the emptiness problem for simple probabilistic co-Büchi word automata with almost-sure semantics to the emptiness problem for universal co-Büchi tree automata with qualitative semantics. The correctness of the reduction relies on the two following results (Lemma 4.3 and Lemma 4.4).

Let $\mathcal{A}=\left(Q, q_{i n}, \delta\right)$ be a simple probabilistic word automaton and $F \subseteq Q$. Define the following probabilistic tree automata:

- $\mathcal{A}_{1}=\left(Q, q_{i n}, \delta^{\prime}\right)$ where $\delta^{\prime}(p, a)=\frac{1}{2}\left(q_{1}, q_{1}\right)+\frac{1}{2}\left(q_{2}, q_{2}\right)$ if $\delta(p, a)=\frac{1}{2} q_{1}+\frac{1}{2} q_{2}$.

- $\mathcal{A}_{2}=\left(Q, q_{i n}, \delta^{\prime \prime}\right)$ where $\delta^{\prime \prime}(p, a)=\frac{1}{2}\left(q_{1}, q_{2}\right)+\frac{1}{2}\left(q_{2}, q_{1}\right)$ if $\delta(p, a)=\frac{1}{2} q_{1}+\frac{1}{2} q_{2}$.

Lemma 4.3 relates $\mathcal{A}_{1}$ and $\mathcal{A}$, where $\mu$ denotes the coin-flipping measure on branches defined in Section 2.5, while Lemma 4.4 relates $\mathcal{A}_{1}$ and $\mathcal{A}_{2}$. 
Lemma 4.3 ([8, Proposition 43]). The following holds:

$$
L_{\text {Qual,co-Büchi(F) }}^{\forall^{=1}}\left(\mathcal{A}_{1}\right)=\left\{t \in \operatorname{Trees}(\Sigma): \mu\left(\left\{b \in\{0,1\}^{\omega}: t[b] \in L_{\text {co-Büchi(F) }}^{=1}(\mathcal{A})\right\}\right)=1\right\} .
$$

Now, for a fixed tree $t$, the Markov chains $\mathcal{M}_{\mathcal{A}_{1}}^{t}$ and $\mathcal{M}_{\mathcal{A}_{2}}^{t}$ associated with $\mathcal{A}_{1}$ and $\mathcal{A}_{2}$ respectively are equal: indeed, they have the same states $Q \times\{0,1\}^{*}$, the same initial state $\left(q_{i n}, \varepsilon\right)$ and the same probability transition function $T$ given by

$$
T((q, u))=\frac{1}{4}\left(q_{1}, u 0\right)+\frac{1}{4}\left(q_{1}, u 1\right)+\frac{1}{4}\left(q_{2}, u 0\right)+\frac{1}{4}\left(q_{2}, u 1\right)
$$

where $\delta(q, t(u))=\frac{1}{2} q_{1}+\frac{1}{2} q_{2}$ in $\mathcal{A}$. As a consequence, $\mathcal{A}_{1}$ and $\mathcal{A}_{2}$ have the same qualitative coBüchi semantics.

LEMMA 4.4.

$$
L_{Q u a l, c o-B u ̈ c h i(F)}^{\forall}\left(\mathcal{A}_{2}\right)=L_{\text {Qual,co-Büchi }(F)}^{\forall=1}\left(\mathcal{A}_{1}\right) .
$$

We are now ready to prove Theorem 4.2.

Proof of Theorem 4.2. Let $\mathcal{A}=\left(Q, q_{i n}, \delta\right)$ be a simple probabilistic word automaton and $F \subseteq Q$. We define the tree automaton $\mathcal{A}_{U}=\left(Q, q_{\text {in }}, \Delta\right)$ where

$$
\Delta=\left\{\left(q, a, q_{1}, q_{2}\right),\left(q, a, q_{2}, q_{1}\right) \mid \delta(q, a)=\frac{1}{2} q_{1}+\frac{1}{2} q_{2}\right\} .
$$

Now, we establish that $L_{\text {co-Büchi }(F)}^{=1}(\mathcal{A}) \neq \emptyset$ if, and only if, $L_{\text {Qual,co-Büchi }(F)}^{\forall}\left(\mathcal{A}_{U}\right) \neq \emptyset$, which concludes the proof of Theorem 4.2.

Assume that there is some $w=w_{0} w_{1} \cdots \in L_{\text {co-Büchi }(F)}^{=1}(\mathcal{A})$, that is such that $P_{\mathcal{A}}^{w}(\operatorname{co-Büchi}(F))=$ 1. We construct a tree $t_{w}$ whose branches are all equal to $w$, i.e. $t_{w}(u)=w_{|u|}$ for every $u \in\{0,1\}^{*}$.

For a fixed run $\rho$ of $\mathcal{A}_{U}$ over $t_{w}$, there is a bijection between the infinite paths of $\mathcal{M}_{\mathcal{A}}^{w}$ and $\mathcal{M}_{\mathcal{A}_{U}}^{\rho}$ that preserves the measure (it suffices to notice that the measure is preserved for cones) and also the property of visiting finitely many states in $F$. As a result, $P_{\mathcal{A}}^{w}(\operatorname{co}-\operatorname{Büchi}(F))=1$ entails $P_{\mathcal{A}_{U}}^{\rho}(\operatorname{co}-\operatorname{Büchi}(F))=1$, for all runs $\rho$. Thus $t_{w} \in L_{\text {Qual,co-Büchi }(F)}^{\forall}\left(\mathcal{A}_{U}\right)$.

The converse implication is not immediate because a tree $t \in L_{\text {Qual,co-Büchi }(F)}^{\forall}\left(\mathcal{A}_{U}\right)$ may not necessarily be of the form $t_{w}$ for some word $w \in \Sigma^{\omega}$.

In Section 2.7, we informally said that an equivalent definition of almost-sure acceptance for probabilistic tree automata can be obtained by associating a probability measure on the set of all runs induced by a tree, and by requiring the measure of the set of qualitatively accepting runs to be equal to 1 ; in this approach the notion of a run is the same as for (non-probabilistic) tree automata (see [8] for details).

Now, consider the probabilistic tree automaton $\mathcal{A}_{2}$ used in Lemma 4.4: for a fixed tree $t$, the set of runs of $\mathcal{A}_{U}$ over $t$ is the same as the set of runs of $\mathcal{A}_{2}$ over $t$. Since all runs of $\mathcal{A}_{U}$ over $t$ are qualitatively accepted, then all runs of $\mathcal{A}_{2}$ over $t$ are qualitatively accepted too, so the set of qualitatively accepting runs of $\mathcal{A}_{2}$ over $t$ has measure 1 . In other words, $t \in L_{\text {Qual,co-Büchi }(F)}^{\forall=1}\left(\mathcal{A}_{2}\right)$. Hence, by Lemma 4.4, $t \in L_{\text {Qual,co-Büchi }(F)}^{\forall=1}\left(\mathcal{A}_{1}\right)$. Finally, using Lemma 4.3, almost all branches of $t$ are in $L_{\text {co-Büchi }(F)}^{=1}(\mathcal{A})$, entailing $L_{\text {co-Büchi }(F)}^{=1}(\mathcal{A}) \neq \emptyset$.

\section{COROLLARIES}

In this section we derive two corollaries from Theorem 4.2: the undecidability of the MSO $+\underset{\text { path }}{=1}$ theory of the infinite binary tree (Theorem 5.1), and the undecidability of the emptiness problem for alternating probabilistic automata with non-zero semantics (Theorem 5.2). 


\subsection{Undecidability of $\mathrm{MSO}+\underset{\text { path }}{=1}$}

Before stating the problem, we refer the reader to [32] for definitions and basic properties on Monadic Second Order logic (MSO) for trees.

The logic MSO $+\forall^{=1}$, introduced and studied in [24, 25], extends MSO with a probabilistic operator $\forall^{=1} X . \varphi$ stating that the set of all sets $X$ satisfying $\varphi$ contains a subset of Lebesgue-measure one. Michalewski, Mio and Skrzypczak proved in these papers that the MSO $+\forall^{=1}$-theory of the infinite binary tree is undecidable. They also considered a variant of this logic, denoted by MSO $+\underset{\text { path }}{=1}$, in which the quantification in the probabilistic operator is restricted to sets of nodes that form a path. They proved that, in terms of expressiveness, $\mathrm{MSO}+\forall_{\text {path }}^{=1}$ is between $\mathrm{MSO}$ and $\mathrm{MSO}+\forall^{=1}$, with a strict gain in expressiveness compared to MSO. However, they left open the question of the decidability of the $\mathrm{MSO}+{ }_{\text {path }}^{=1}$ theory of the infinite binary tree [25, Problem 4].

In this section, we establish that in fact $M S O+\forall_{\text {path }}^{=1}$ is undecidable over the infinite binary tree, as a direct consequence of Theorem 4.2.

The syntax of $\mathrm{MSO}+\underset{\text { path }}{=1}$ is given by the following grammar:

$$
\varphi::=\operatorname{succ}_{0}(x, y)\left|\operatorname{succ}_{1}(x, y)\right| x \in X|\neg \varphi| \varphi_{1} \wedge \varphi_{2}|\forall x . \varphi| \forall X . \varphi \mid \forall_{\text {path }}^{=1} X . \varphi
$$

where $x$ ranges over a countable set of first-order variables, and $X$ ranges over a countable set of monadic second-order variables (also called set variables). The quantifier $\underset{\text { path }}{\forall=1}$ is called the path-

\section{measure quantifier.}

The semantics of MSO on the infinite binary tree is defined by interpreting the first-order variables $x$ as nodes, and the set variables $X$ as subsets of nodes. Ordinary quantification and the Boolean operations are defined as usual, $x \in X$ is interpreted as the membership relation, and $\operatorname{succ}_{i}$ (for $i=0,1$ ) is interpreted as the binary relation $\left\{(x, x \cdot i) \mid x \in\{0,1\}^{*}\right\}$. We now describe how to interpret quantified formulas of the form $\forall_{\text {path }}^{=1} X . \varphi$. A path is a prefix-closed non-empty set $X \subseteq\{0,1\}^{*}$ such that for any node $v \in X$ either $v 0 \in X$ or $v 1 \in X$, but not both. We let Paths denote the set of all paths. Note that there is a one-to-one correspondence between Paths and the set $\{0,1\}^{\omega}$ of branches. Thus, the coin-flipping measure $\mu$, defined over $\{0,1\}^{\omega}$ (see Section 2.5), induces a measure over Paths, which we write $\mu$. We let $t \vDash \underset{\text { path }}{=1} X . \varphi$ if there exists a measurable subset of paths $\Pi \subseteq$ Paths with $\mu(\Pi)=1$ and such that for any $\pi \in \Pi$ one has $t, \pi \vDash \varphi$.

A sentence is a formula without free variables. The $\mathbf{M S O}+\underset{\text { path }}{\forall}=1$-theory of the infinite binary tree is the set of all $\mathrm{MSO}+\mathrm{V}_{\text {path }}^{=1}$-sentences $\varphi$ that hold in the infinite binary tree.

We identify a $\{0,1\}^{n}$-tree $t$ with a tuple of $n$ subsets of nodes setTuple $(t)=\left(X_{1}, \ldots, X_{n}\right)$ where a node $x$ belongs to $X_{i}$ if and only if the $i$-th element of $t(x)$ is 1 . This immediately permits to interpret an $\mathrm{MSO}+\underset{\text { path }}{\forall}=1$ formula with $n$ free set variables on $\{0,1\}^{n}$-trees.

The following result is an easy consequence of Theorem 4.2.

THEOREM 5.1. The MSO $+\underset{\text { path }}{=1}$-theory of the infinite binary tree is undecidable.

Proof. We reduce the emptiness problem for co-Büchi tree automata with qualitative semantics, that we proved undecidable (Theorem 4.2), to the $\mathrm{MSO}+\underset{\text { path }}{=1}$-theory of the infinite binary tree.

Let $\mathcal{A}$ be a co-Büchi tree automaton over the alphabet $\Sigma$. Without loss of generality, we assume that $\Sigma \subseteq\{0,1\}^{n}$ for some $n$. Note that, as MSO $+\forall_{\text {path }}^{=1}$-formulas are interpreted over the (unlabelled) infinite binary tree, we use tuples of subsets of nodes to encode $\Sigma$-trees. We construct an MSO $+\underset{\text { path }}{\forall}=1$ formula $\varphi(\vec{X})$, with $\vec{X}=\left(X_{1}, \ldots, X_{n}\right)$, such that

$$
L_{\text {Qual,co-Büchi }(F)}^{\forall}(\mathcal{A})=\{t \mid \operatorname{set} \operatorname{Tuple}(t) \vDash \varphi\} .
$$


The formula $\varphi$ mimics the definition of $L_{\text {Qual,co-Büchi }(F)}^{\forall}(\mathcal{A})$ :

$$
\forall \vec{Y} \text {.(" } \vec{Y} \text { is a run of } \mathcal{A} \text { on } \vec{X} \text { " } \Rightarrow \forall_{\text {path }}^{=1} Z \text {. (" } Z \text { is an accepting path of } \vec{Y} \text { ")), }
$$

where " $\vec{Y}$ is a run of $\mathcal{A}$ on $\vec{X}$ " and " $Z$ is an accepting path of $\vec{Y}$ " are expressed in first-order logic (we refer to [29] for this classical encoding). The desired formula is then $\neg \exists \vec{X} \varphi$, which achieves the proof.

\subsection{Undecidability of the Emptiness Problem for Alternating Tree Automata with Non-zero Semantics}

The non-zero semantics for tree automata was introduced by Bojańczyk, Gimbert and Kelmendi [6]. In a recent paper, Fournier and Gimbert initiated the study of alternating tree automata with nonzero semantics [19]. Their main result is the decidability of the emptiness problem for a subclass of these automata, called limited choice for Abélard, and this is used to solve the satisfiability problem of $\mathrm{CTL}^{*}+\mathrm{pCTL}$; however the decidability of emptiness for the full class of alternating automata with non-zero semantics was left open. Since this class easily subsumes universal tree automata with qualitative semantics, Theorem 4.2 directly implies that this problem is undecidable.

An alternating non-zero automaton on alphabet $\Sigma$ is a tuple

$$
\mathcal{A}=\left((Q, \prec), q_{\text {in }}, Q_{E}, Q_{A}, \Delta, F_{\forall}, F_{1}, F_{>0}\right)
$$

where $Q$ is a finite set of states equipped with a total order $\prec, q_{\text {in }} \in Q$ is the initial state, $\left(Q_{E}, Q_{A}\right)$ is a partition of $Q$ into Éloïse's and Abélard's states, $\Delta$ is a set of transitions made of local transitions (elements of $Q \times \Sigma \times Q$ ) and split transitions (elements of $Q \times \Sigma \times Q \times Q$ ), and $F_{\forall}, F_{1}, F_{>0} \subseteq Q$ are subsets of $Q$ defining the semantics of the acceptance game $\mathcal{G}_{\mathcal{A}, t}^{\mathrm{n}-\mathrm{Z}}$, to be defined later.

The input of such an automaton is a $\sum$-tree $t$ and acceptance is defined thanks to a two-player perfect-information stochastic game. The arena is quite similar to the arena $\mathcal{G}_{\mathcal{A}, t}^{=1}$ defined in Section 2.6 for alternating tree automata with qualitative semantics (simply ignore the total order < and subsets $F_{\forall}, F_{1}, F_{>0}$ ), except that local transitions are handled without interacting with the Random player (i.e. when Éloïse or Abélard simulates a local transition the state is simply updated and the pebble stays in the same node).

Formally one lets $G=\left(V_{E} \cup V_{A} \cup V_{R}, E\right)$ with $V_{E}=Q_{E} \times\{0,1\}^{*}, V_{A}=Q_{A} \times\{0,1\}^{*}$ and $V_{R}=$ $\left\{\left(q, u, q_{0}, q_{1}\right) \mid u \in\{0,1\}^{*}\right.$ and $\left.\left(q, t(u), q_{0}, q_{1}\right) \in \Delta\right\}$, and

$$
\begin{aligned}
E=\quad & \left.\left\{\left((q, u),\left(q^{\prime}, u\right)\right) \mid\left(q, t(u), q^{\prime}\right) \in \Delta\right)\right\} \cup \cup \\
& \left.\left\{\left((q, u),\left(q, u, q_{0}, q_{1}\right)\right) \mid\left(q, t(u), q_{0}, q_{1}\right) \in \Delta\right)\right\} \quad \cup \\
& \left.\left\{\left(\left(q, u, q_{0}, q_{1}\right),\left(q_{x}, u \cdot x\right)\right) \mid x \in\{0,1\} \text { and }\left(q, u, q_{0}, q_{1}\right) \in V_{R}\right)\right\}
\end{aligned}
$$

Then, we define $\mathcal{G}_{\mathcal{A}, t}^{\mathrm{n}-\mathrm{z}}=\left(G, V_{E}, V_{A}, V_{R}, \delta,\left(q_{i n}, \varepsilon\right)\right)$ where $\delta\left(\left(q, u, q_{0}, q_{1}\right)\right)=\frac{1}{2}\left(q_{0}, u 0\right)+\frac{1}{2}\left(q_{1}, u 1\right)$. A strategy $\sigma_{E}$ for Éloïse beats a strategy for Abélard $\sigma_{A}$ if all the following conditions are satisfied:

(i) Sure winning: in every play consistent with $\left(\sigma_{E}, \sigma_{A}\right)$ the largest (with respect to $\prec$ ) state appearing infinitely often belongs to $F_{\forall}$.

(ii) Almost-sure winning: the (measurable) set of plays consistent with $\left(\sigma_{E}, \sigma_{A}\right)$ where the largest state (with respect to $\prec$ ) appearing infinitely often belongs to $F_{1}$ has measure 1 .

(iii) Positive winning: for every history consistent with $\left(\sigma_{E}, \sigma_{A}\right)$ that ends with a state in $F_{>0}$, the (measurable) set of infinite continuations of this history that contain only states in $F_{>0}$ and are consistent with $\left(\sigma_{E}, \sigma_{A}\right)$, has non-zero measure. 
Finally, a tree $t$ is accepted by $\mathcal{A}$ if, and only if, Éloïse has a strategy that beats any strategy of Abélard. The emptiness problem asks for a given alternating non-zero automaton whether the set of accepted trees is empty.

It is easily seen that alternating automata with non-zero semantics subsume universal co-Büchi tree automata with qualitative semantics. Indeed, consider a universal co-Büchi tree automaton $\mathcal{A}$ with qualitative semantics having a set of states $Q$ and a set of states $F \subseteq Q$ defining the co-Büchi condition. Then, universality is captured by alternation (see Proposition 2.9) and the co-Büchi qualitative acceptance condition of $\mathcal{A}$ can be expressed by part (ii) of the beating condition: it is enough to rank the states in $F$ higher than those in $Q \backslash F$ in the total order on $Q$, and to let $F_{1}=Q \backslash F$.

Together with Theorem 4.2 this yields the following undecidability result.

THEOREM 5.2. The emptiness problem for alternating tree automata with non-zero semantics is undecidable.

Proof. Consider a co-Büchi universal tree automaton $\mathcal{A}=\left(Q, q_{i n}, \Delta\right)$ whose acceptance condition is given by a subset $F \subseteq Q$. Without loss of generality, we can safely assume that $Q=$ $\left\{q_{1}, \ldots q_{n}\right\}$ where $n=|Q|$ and that $F=\left\{q_{k}, \ldots, q_{n}\right\}$ for some $k \leq n+1$. We construct an alternating non-zero automaton $\mathcal{B}=\left((Q, \prec), q_{\text {in }}, \emptyset, Q, \Delta, Q, F_{1}, Q\right)$, where the total order $<$ on $Q$ is defined by $q_{i}<q_{j}$ if and only if $i<j$ and $F_{1}=Q \backslash F$.

Note that since $\mathcal{A}$ has only split transitions, the arenas $\mathcal{G}_{\mathcal{A}, t}^{=1}$ and $\mathcal{G}_{\mathcal{B}, t}^{\mathrm{n}-\mathrm{z}}$ are the same for any tree $t$, and so are the strategies for Éloïse and Abélard. Moreover, it is immediate that an Éloïse's strategy $\sigma_{E}$ beats an Abélard's strategy $\sigma_{A}$ in $\mathcal{G}_{\mathcal{B}, t}^{\mathrm{n}-\mathrm{z}}$ if, and only if, almost all plays in $\mathcal{G}_{\mathcal{A}, t}^{=1}$ consistent with $\left(\sigma_{E}, \sigma_{A}\right)$ satisfy the co-Büchi condition. Hence, Éloïse has a strategy that beats any strategy of Abélard in $\mathcal{G}_{\mathcal{B}, t}^{\mathrm{n}-\mathrm{z}}$ if and only if she has an almost-surely winning strategy in the co-Büchi game $\left(\mathcal{G}_{\mathcal{A}, t}^{=1}, F \times\{0,1\}^{*}\right)$. Otherwise said, a tree is accepted by $\mathcal{B}$ if, and only if, it is accepted by $\mathcal{A}$.

Applying Theorem 4.2, concludes the proof.

\section{CONCLUSIONS}

The core contribution is the study of alternating automata with qualitative semantics and the identification of a sharp decidability frontier for their emptiness problem: the emptiness problem is decidable for Büchi objectives, but it is undecidable for the co-Büchi objectives. The latter undecidability result directly implies the undecidability of $\mathrm{MSO}+\underset{\text { path }}{\forall}=1$ in an elegant manner. In an attempt to exhibiting a decidable extension of MSO with a probabilistic operator, a natural track is to seek natural subclasses of alternating tree automata with qualitative semantics (or even of non-zero automata) with a decidable emptiness problem. However, while for alternating Buchi tree automata with qualitative semantics emptiness problem is decidable, their connection with a robust logic is unclear. The recent results concerning restrictions to thin quantification $[5,6]$ and to limited choice for Abélard [19] bring hope and inspiration for the construction of such subclasses.

\section{REFERENCES}

[1] Christel Baier, Marcus Größer, and Nathalie Bertrand. 2012. Probabilistic $\omega$-Automata. f. ACM 59, 1 (2012), 1.

[2] Vince Bárány, Łukasz Kaiser, and Alex Rabinovich. 2010. Expressing Cardinality Quantifiers in Monadic Second-Order Logic over Trees. Fundamenta Informaticae 100, 1-4 (2010), 1-17.

[3] Raphaël Berthon, Emmanuel Filiot, Shibashis Guha, Bastien Maubert, Nello Murano, Laureline Pinault, Jean-François Raskin, and Sasha Rubin. 2019. Monadic Second-Order Logic with Path-Measure Quantifier is Undecidable. CoRR abs/1901.04349 (2019).

[4] Nathalie Bertrand, Blaise Genest, and Hugo Gimbert. 2009. Qualitative Determinacy and Decidability of Stochastic Games with Signals. In Proceedings of the 24th Annual IEEE Symposium on Logic in Computer Science. IEEE, 319-328. 
[5] Mikołaj Bojańczyk. 2016. Thin MSO with a Probabilistic Path Quantifier. In Proceedings of the 43rd International Colloquium on Automata, Languages, and Programming (LIPIcs, Vol. 55). Schloss Dagstuhl - Leibniz-Zentrum fuer Informatik, 96:1-96:13.

[6] Mikołaj Bojańczyk, Hugo Gimbert, and Edon Kelmendi. 2017. Emptiness of Zero Automata Is Decidable. In Proceedings of the 44th International Colloquium on Automata, Languages, and Programming (LIPIcs, Vol. 80). Schloss Dagstuhl Leibniz-Zentrum fuer Informatik, 106:1-106:13.

[7] Mikołaj Bojańczyk, Edon Kelmendi, and Michal Skrzypczak. 2019. MSO+ $\nabla$ is Undecidable. In Proceedings of the 34th Annual ACM/IEEE Symposium on Logic in Computer Science. IEEE, 1-13.

[8] Arnaud Carayol, Axel Haddad, and Olivier Serre. 2014. Randomization in Automata on Infinite Trees. ACM Transactions on Computational Logic 15, 3 (2014), 24:1-24:33.

[9] Arnaud Carayol, Christof Löding, and Olivier Serre. 2018. Pure Strategies in Imperfect Information Stochastic Games. Fundamenta Informaticae 160, 4 (2018), 361-384.

[10] Krishnendu Chatterjee and Laurent Doyen. 2014. Partial-Observation Stochastic Games: How to Win when Belief Fails. ACM Transactions on Computational Logic 15, 2 (2014), 16:1-16:44.

[11] Krishnendu Chatterjee, Laurent Doyen, Thomas A. Henzinger, and Jean-François Raskin. 2007. Algorithms for OmegaRegular Games with Imperfect Information. Logical Methods in Computer Science 3, 3 (2007).

[12] Costas Courcoubetis and Mihalis Yannakakis. 1990. Markov Decision Processes and Regular Events (Extended Abstract). In Proceedings of the 17th International Colloquium on Automata, Languages, and Programming (ICALP 1990) (Lecture Notes in Computer Science, Vol. 443). Springer, 336-349.

[13] Luca de Alfaro. 1999. The Verification of Probabilistic Systems Under Memoryless Partial-Information Policies is Hard. In Proceedings of the 2nd International Workshop on Probabilistic Methods in Verification. 19-32.

[14] Ronald Fagin, Joseph Y. Halpern, Yoram. Moses, and Moshe Y. Vardi. 1995. Reasoning about Knowledge. MIT Press.

[15] Nathanaël Fijalkow. 2017. Undecidability Results for Probabilistic Automata. SIGLOG News 4, 4 (2017), 10-17.

[16] Nathanaël Fijalkow, Hugo Gimbert, Edon Kelmendi, and Youssouf Oualhadj. 2015. Deciding the Value 1 Problem for Probabilistic Leaktight Automata. Logical Methods in Computer Science 11, 2 (2015), 1-42.

[17] Nathanaël Fijalkow, Sophie Pinchinat, and Olivier Serre. 2013. Emptiness of Alternating Tree Automata Using Games with Imperfect Information. In Proceedings of IARCS Annual Conference on Foundations of Software Technology and Theoretical Computer Science (LIPIcs, Vol. 24). Schloss Dagstuhl - Leibniz-Zentrum fuer Informatik, 299-311.

[18] Nathanaël Fijalkow, Sophie Pinchinat, and Olivier Serre. 2013. Emptiness of Alternating Tree Automata Using Games with Imperfect Information. (2013). https://hal.inria.fr/hal-01260682

[19] Paulin Fournier and Hugo Gimbert. 2018. Alternating Nonzero Automata. In Proceedings of the 29th International Conference on Concurrency Theory (LIPIcs, Vol. 118). Schloss Dagstuhl - Leibniz-Zentrum fuer Informatik, 13:1-13:16.

[20] Hugo Gimbert and Youssouf Oualhadj. 2010. Probabilistic Automata on Finite Words: Decidable and Undecidable Problems. In Proceedings of the 37th International Colloquium on Automata, Languages and Programming (Lecture Notes in Computer Science, Vol. 6199). Springer, 527-538.

[21] Hugo Gimbert and Wiesław Zielonka. 2007. Perfect Information Stochastic Priority Games. In Proceedings of the 34th International Colloquium on Automata, Languages, and Programming (Lecture Notes in Computer Science, Vol. 4596). Springer, 850-861.

[22] Vincent Gripon and Olivier Serre. 2009. Qualitative Concurrent Stochastic Games with Imperfect Information. In Proceedings of the 36th International Colloquium on Automata, Languages, and Programming (Lecture Notes in Computer Science, Vol. 5556). Springer, 200-211.

[23] Antonín Kučera. 2011. Turn-Based Stochastic Games. In Lectures in Game Theory for Computer Scientists, Krzysztof R. Apt and Erich Grdel (Eds.). Cambridge University Press, New York, NY, USA, Chapter 5, 146-184.

[24] Henryk Michalewski and Matteo Mio. 2016. Measure Quantifier in Monadic Second Order Logic. In Proceedings of Logical Foundations of Computer Science - International Symposium (Lecture Notes in Computer Science, Vol. 9537). Springer, 267-282.

[25] Matteo Mio, Michał Skrzypczak, and Henryk Michalewski. 2018. Monadic Second Order Logic with Measure and Category Quantifiers. Logical Methods in Computer Science 14, 2 (2018).

[26] Azaria Paz. 1971. Introduction to Probabilistic Automata. Academic Press.

[27] Martin L. Puterman. 1994. Markov Decision Processes: Discrete Stochastic Dynamic Programming. John Wiley \& Sons, Inc., New York, NY, USA.

[28] Michael O. Rabin. 1963. Probabilistic Automata. Information and Control 6, 3 (1963), 230-245.

[29] Michael O. Rabin. 1969. Decidability of Second-Order Theories and Automata on Infinite Trees. Transactions of the AMS 141 (1969), 1-35.

[30] J.H. Reif. 1979. Universal Games of Incomplete Information. In Proc of STOC'79. ACM, 288-308.

[31] J.H. Reif. 1984. The Complexity of Two-Player Games of Incomplete Information. 7. Comput. System Sci. 29, 2 (1984), 274-301. 
[32] Wolfgang Thomas. 1997. Languages, Automata, and Logic. In Handbook of Formal Language Theory, G. Rozenberg and A. Salomaa (Eds.). Vol. III. 389-455.

[33] Wiesław Zielonka. 1998. Infinite Games on Finitely Coloured Graphs with Applications to Automata on Infinite Trees. Theoretical Computer Science 200, 1-2 (1998), 135-183. 